\title{
KamLAND-Experiment and Soliton-Like Nuclear Georeactor. Part 1. Comparison of Theory with Experiment
}

\author{
V. D. Rusov ${ }^{1}$, D. A. Litvinov ${ }^{1}$, E. P. Linnik ${ }^{1}$, V. N. $\operatorname{Vaschenko}^{2}$, T. N. Zelentsova ${ }^{1}$, \\ M. E. Beglaryan ${ }^{1}$, V. A. Tarasov ${ }^{1}$, S. A. Chernegenko', V. P. Smolyar ${ }^{1}$, \\ P. O. Molchinikolov ${ }^{1}$, K. K. Merkotan ${ }^{1}$, P. E. Kavatskyy ${ }^{3}$ \\ ${ }^{1}$ Department of Theoretical and Experimental Nuclear Physics, \\ Odessa National Polytechnic University, Odessa, Ukraine \\ ${ }^{2}$ State Ecological Academy for Postgraduate Education and Management, Kyiv, Ukraine \\ ${ }^{3}$ Research Institute of Sustainable Development Technologies "Live Earth", Kyiv, Ukraine \\ Email: "siiis@te.net.ua
}

Received December 2, 2012; revised January 5, 2013; accepted February 1, 2013

Copyright (C) 2013 V. D. Rusov et al. This is an open access article distributed under the Creative Commons Attribution License, which permits unrestricted use, distribution, and reproduction in any medium, provided the original work is properly cited.

\begin{abstract}
We give an alternative description of the data produced in the KamLAND experiment, assuming the existence of a natural nuclear reactor on the boundary of the liquid and solid phases of the Earth core. Analyzing the uncertainty of antineutrino spectrum of georeactor origin, we show that the theoretical (which takes into account the soliton-like nuclear georeactor) total reactor antineutrino spectra describe with good accuracy the experimental KamLAND-data over the years of 2002-2007 and 2002-2009, respectively. At the same time, the parameters of mixing $\left(\Delta m_{21}^{2}=2.5 \times 10^{-5} \mathrm{eV}^{2}\right.$, $\left.\tan ^{2} \theta_{12}=0.437\right)$ calculated within the framework of georeactor hypothesis substantially differ from the parameters of mixing $\left(\Delta m_{21}^{2}=7.49 \times 10^{-5} \mathrm{eV}^{2}, \tan ^{2} \theta_{12}=0.436\right)$ obtained in KamLAND-experiment for total exposure over the period of 2002-2009. By triangulation of KamLAND and Borexino data we have constructed the coordinate location of soliton-like nuclear georeactors on the boundary of the liquid and solid phases of the Earth core.
\end{abstract}

Keywords: KamLAND Experiment; Borexino Experiment; Nuclear Georeactor; Neutrino Oscillations; Parameters of Mixing

\section{Introduction}

It is obvious now that the experiments by the KamLAND-collobaration over the last 8 years [1-5] are extremely important not only for observation of reactor antineutrino oscillations, but because they make it possible for the first time to verify one of the most vivid and mysterious ideas in nuclear geophysics - the hypothesis of natural nuclear georeactor existence [6-20]. In spite of its singularity and long history, this hypothesis becomes especially attractive today because it lets one explain clearly from the physical standpoint different unrelated, at the first glance, geophysical anomalous phenomena the fundamental nature of which is beyond any doubt.

First of all it concerns the problem of ${ }^{3} \mathrm{He}$ and ${ }^{4} \mathrm{He}$ isotopes origin in the Earth interior, the concentration ratio

"Corresponding author. of which, as is well known, "mystically" increases towards the center of Earth $[21,22]$. This is practically impossible to explain by existing models of the origin of the anomalous ${ }^{3} \mathrm{He}$ concentration and ${ }^{3} \mathrm{He} /{ }^{4} \mathrm{He}$ ratio distribution in the Earth interior since they have serious contradictions. For example, Anderson et al. has pointed out [23]: "The model whereby high ${ }^{3} \mathrm{He} /{ }^{4} \mathrm{He}$ is attributed to a lower mantle source, and is thus effectively an indicator of plumes, is becoming increasingly untenable as evidence for a shallow origin for many high ${ }^{3} \mathrm{He} /{ }^{4} \mathrm{He}$ hot spots accumulates. Shallow, low ${ }^{4} \mathrm{He}$ for high ${ }^{3} \mathrm{He} /{ }^{4} \mathrm{He}$ are logically reasonable, cannot be ruled out, and need to be rigorously tested if we are to be understand the full implications of this important geochemical tracer". Apparently, the most advanced model, which is devoid of the mentioned contradictions, is the so-called Gonnermann-Mukhopadhyay model, preserving noble gases in convective 
mantle [24]. However this model ignores the possible high concentrations of ${ }^{238} \mathrm{U}$ and ${ }^{232} \mathrm{Th}$ in the outer core (as it is shown by numerous laboratory experiments [16, $18,20]$ ), and this is the weak point of this model. At the same time, it is shown [17] that, if the nuclear georeactor exists, within the framework of model, which takes into account the georeactor thermal power and distribution of ${ }^{238} \mathrm{U}$ and ${ }^{232} \mathrm{Th}$ in the Earth interior, it is possible also to obtain a good description of the known experimental ${ }^{3} \mathrm{He} /{ }^{4} \mathrm{He}$ distributions in the crust and mantle.

A potent argument in favour of the nuclear georeactor existence are the results of recent seismo-tomography researches of the anomalous high heat flow $(13 \pm 4$ TW $)$ on the core-mantle boundary. This heat is much higher than the radiogenic heat in the lower mantle ( $D^{\prime \prime}$-region) [25]. To explain such an anomalous high heat flow the authors of this paper have advanced the hypothesis of young solid core of the Earth with its crystallization energy as a cause of anomalous temperature effect.

In full measure it concerns the known problem of nature of an energy source maintaining the convection in the Earth liquid core or, more precisely, the mechanism of magneto-hydrodynamic dynamo generating the Earth magnetic field. It is obvious, that the well-known ${ }^{40} \mathrm{~K}$ mechanism of radiogenic heat production in the solid core of the Earth does not solve the problem on the whole, because it can not explain the heat flows balance on the core-mantle boundary (see [26] and refs therein). It is also worth mentioning the so-called mechanism of the Earth magnetic field inversions closely associated with the problem of convection in the Earth liquid core. It seems to be strange, but both these fundamental problems have a simple and physically clear solution within the framework of hypothesis of existence of the natural nuclear georeactor on the boundary of the liquid and solid phases of the Earth [17,27].

If the georeactor hypothesis is true, the fluctuations of georeactor thermal power can influence on Earth global climate in the form of anomalous temperature jumps in the following way. Strong fluctuations of georeactor thermal power can lead to the partial blocking of convection in the liquid core [27] and the change of an angular velocity of liquid geosphere rotation, thereby, by virtue of a conservation law of Earth angular moment to the change of angular velocity of mantle and the Earth surface, respectively. It means that the heat or, more precisely, dissipation energy caused by friction of Earth surface and bottom layer can make a considerable contribution to total energy balance of the atmosphere and thereby to influence significantly on the Earth global climate evolution [27].

However, in spite of the obvious attractiveness of this hypothesis there are some difficulties for its perception predetermined by non-trivial properties which georeactor must possess. At first, natural, i.e. unenriched, uranium or thorium must be used as a nuclear fuel. Secondly, tra- ditional control rods are completely absent in the reactivity regulation system of reactor. Thirdly, in spite of the absence of control rods a reactor must possess the property of so-called inner safety. It means that the critical state of reactor core must be permanently maintained in any situation, i.e. the normal operation of reactor is automatically maintained not as a result of operator activity, but by virtue of physical reasons-laws preventing the explosive development of chain reaction in a natural way. Figuratively speaking, the reactor with inner safety is the "nuclear installation which never explodes" [28].

It seems to be strange, but reactors satisfying such unusual requirements are possible in reality. For the first time the idea of such a self-regulating fast reactor (socalled mode of breed-and-burn) was expressed in a general form at II Genevan conference in 1958 by Russian physicists Feynberg and Kunegin [29] and relatively recently "reanimated" as an idea of the self-regulating fast reactor in traveling-wave mode of nuclear burning by $\mathrm{L}$. Feoktistov [30] and independently by Teller, Ishikawa and Wood [31].

To interpret the experimental KamLAND antineutrino spectra [3-5] we consider below the properties of such an unusual reactor.

\section{Soliton-Like Nuclear Georeactor and the KamLAND Antineutrino Spectra (Experiments over the Period of 2002-2004)}

The main idea of reactor with inner safety consists in selection of fuel composition so that, at first, the characteristic time $\tau_{\beta}$ of nuclear burning of fuel active (fissile) component is substantially greater than the characteristic time of delayed neutrons production and, secondly, necessary self-regulation conditions are fulfilled during the reactor operation (that always take place when the equilibrium concentration of fuel active component is greater than its critical concentration [30]). These very important conditions can practically always be attained, if among other reactions in a reactor core the chain of nuclear transformations of the Feoktistov uranium-plutonium cycle type [30]

$$
\begin{aligned}
& { }^{238} \mathrm{U}(n, \gamma) \longrightarrow{ }^{239} \mathrm{U} \stackrel{\beta-}{\longrightarrow}{ }^{239} \mathrm{~Np} \\
& \stackrel{\beta-}{\longrightarrow}{ }^{239} \mathrm{Pu}(n, \text { fission })
\end{aligned}
$$

or the Teller-Ishikawa-Wood thorium-uranium cycle type [31]

$$
\begin{aligned}
& { }^{232} \mathrm{Th}(n, \gamma) \longrightarrow{ }^{233} \mathrm{Th} \stackrel{\beta-}{\longrightarrow}{ }^{233} \mathrm{~Pa} \\
& \stackrel{\beta-}{\longrightarrow}{ }^{233} \mathrm{U}(n, \text { fission })
\end{aligned}
$$

are appreciable enough.

In both cases the active components of nuclear fuel are the generated fissile isotopes of ${ }^{239} \mathrm{Pu}(1)$ or ${ }^{233} \mathrm{U}$ (1). The 
characteristic time of such a reaction, i.e. the respective $\beta$-decay time, is approximately equal to $\tau_{\beta}=2.3 / \ln 2 \approx 3.3$ days and $\tau_{\beta} \approx 39.5$ days for reactions (1) and (2), respectively. This is several orders of magnitude greater than the time of delayed neutrons production.

Self-regulation of nuclear burning process (under indicated above relation between the equilibrium and critical concentrations of fuel active components [30]) takes place because such a system, being left by itself, cannot pass from a critical state to reactor runaway mode as the critical concentration is bounded above by the finite value of plutonium equilibrium concentration, i.e. $\tilde{n}_{\mathrm{Pu}}>$ $n_{\text {crit }}$. On phenomenological level the self-regulation of nuclear burning is manifested as follows. Increase of a neutron flux due to some reasons will result in rapid burn-up, for example, of plutonium, i.e. in decrease of its concentration, and therefore in decrease of neutron flux, while the new nuclei of ${ }^{239} \mathrm{Pu}$ are produced with the same rate during $\tau_{\beta}=3.3$ days. And vice versa, if the neutron flux is sharply decreased due to external action, the burnup rate decreases too and the plutonium accumulation rate will be increased as well as the number of neutrons produced in a reactor after approximately same time. Analogous situation will be observed for the thorium- uranium cycle (2), but in time $\tau_{\beta}=39.5$ days

The details of the system of kinetic equations for components of nuclear fuel and neutrons (as a diffusion approximation) in such chains are rather simple and were described in detail in our paper [17]. Typical solutions for such a problem have the form of soliton-like concentration wave of nuclear fuel components and neutrons (Equations (3)-(9) in [17]) and are shown in Figure 1. Within the framework of soliton-like fast reactor theory it is easy to show that the phase velocity $u$ of nuclear burning is determined by following approximate equality [32]

$$
\begin{aligned}
& \frac{u \tau_{\beta}}{2 L} \simeq\left(\frac{8}{3 \sqrt{\pi}}\right)^{6} a^{4} \exp \left(-\frac{64}{9 \pi} a^{2}\right), \\
& a^{2}=\frac{\pi^{2}}{4} \cdot \frac{n_{c r i t}}{\tilde{n}_{\text {fis }}-n_{\text {crit }}}
\end{aligned}
$$

where $\tilde{n}_{f i s}$ and $n_{\text {crit }}$ are the equilibrium and critical concentrations of active (fissile) isotope, respectively; $L$ is the average diffusion distance for neutron, $\tau_{\beta}$ is the delay time caused by active (fissile) isotope production, which is equal to the effective period of intermediate nuclei $\beta$-decay in the uranium-plutonium cycle (1) or
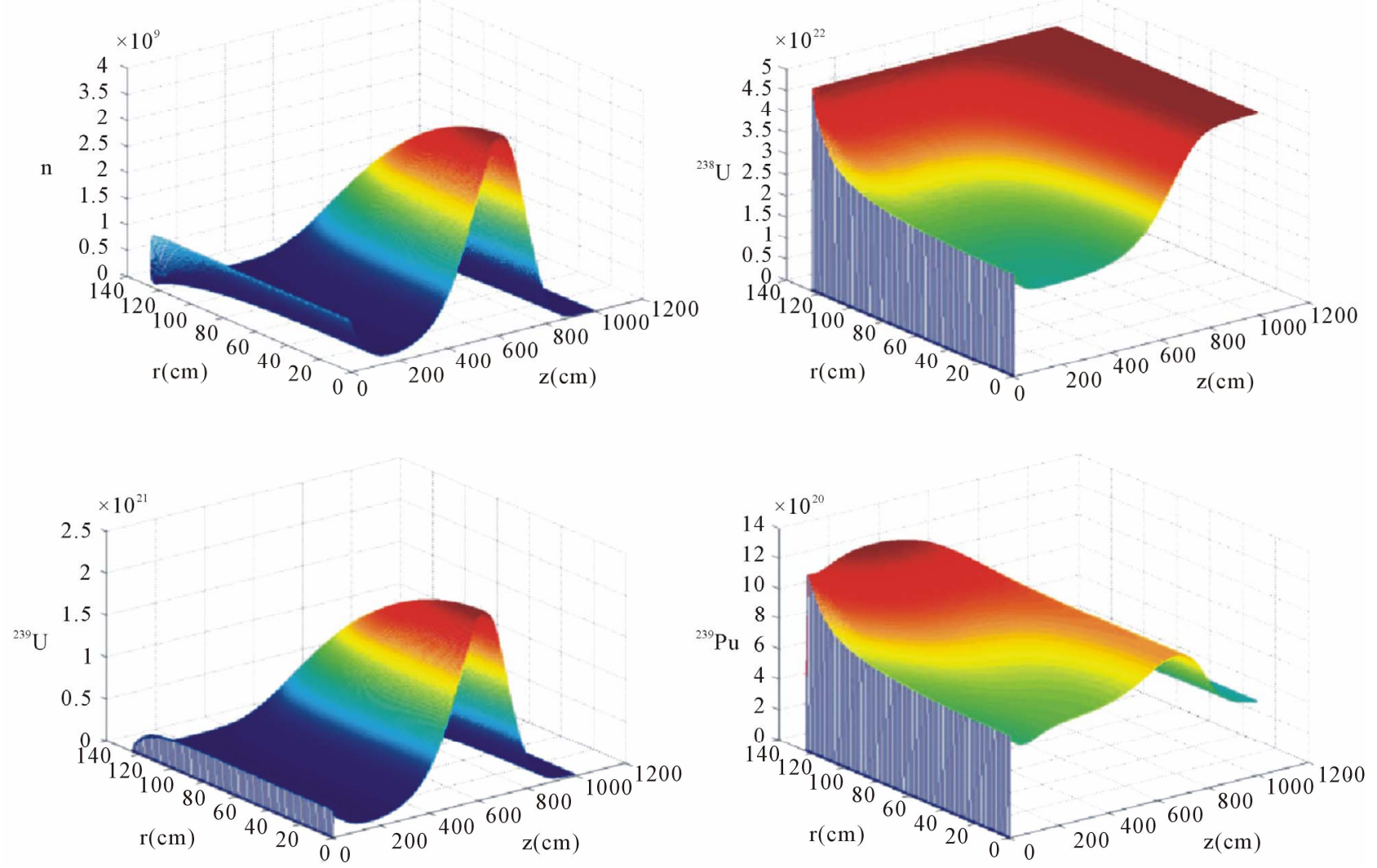

Figure 1. Concentration kinetics of neutrons, ${ }^{238} \mathrm{U},{ }^{239} \mathrm{U},{ }^{239} \mathrm{Pu}$ in the core of cylindrical reactor with radius of $125 \mathrm{~cm}$ and $1000 \mathrm{~cm}$ long at the time of 240 days. Here $r$ is the transverse spatial coordinate axis (cylinder radius), $z$ is the longitudinal spatial coordinate axis (cylinder length). 
thorium-uranium cycle (2).

Note that Equation (3) automatically contains the selfregulation condition for nuclear burning because the existence of a wave is predetermined by the inequality $\tilde{n}_{\text {fis }}>n_{\text {crit }}$ In other words, Equation (3) is a necessary physical requirement for the existence of soliton-like neutron wave of nuclear burning. We indicate for a comparison that, as it follows from Equation (3), the upper bounds of phase velocity of nuclear burning wave are $3.70 \mathrm{~cm} /$ day for the uranium-plutonium cycle (1) and $0.31 \mathrm{~cm} /$ day for the thorium-uranium cycle (2) at almost equal average diffusion distance $(L \sim 5 \mathrm{~cm})$ for fast neutrons $(1 \mathrm{MeV})$ both for uranium and thorium.

Finally, we consider some important details and properties of such a soliton-like fast reactor, assuming the existence of which, we have obtained the theoretical spectra of reactor antineutrino and terrestrial antineutrino which are in good agreement with the experimental KamLAND data [17] corresponding to the first [1] and third [3] exposures.

According to our notions, a soliton-like fast reactor is located on the boundary of the liquid and solid phases of the Earth [17]. The average thickness of such a shellboundary with increased density and mosaic structure is $\sim 2.2 \mathrm{~km}$ [33]. In our opinion, the most advanced mechanism for formation of such a shell below the mantle so far follows from the experimental results by Anisichkin et al. [16,18] and Xuezhao-Secco [34]. According to these results, the chemically stable high-density actinide compounds (particularly uranium carbides and uranium dioxides) lose most of their lithophilic properties at high pressure, sink together with melted iron and concentrate in the Earth core as a consequence of the initial gravitational differentiation of the planet. In the other words, during early stages of the evolution of the Earth and other planets, $\mathrm{U}$ and Th oxides and carbides (as the most dense, refractory, and marginally soluble at high pressures) accumulated from a magma "ocean" on the solid inner core of the planet, thereby activating chain nuclear reactions, and, in particular, a progressing wave of Feoktisov and/or Teller-Ishikawa-Wood type.

What is the thermal power of such a reactor? As a natural quantitative criterion of the georeactor thermal power we used the well-known (based on the geochemical measurements) ${ }^{3} \mathrm{He} /{ }^{4} \mathrm{He}$ radial distribution in the Earth interior [17]. It turned out that the experimental average values of ${ }^{3} \mathrm{He} /{ }^{4} \mathrm{He}$ for crust, the depleted upper mantle, the mantle (minus the depleted upper mantle) and the so called $D^{\prime \prime}$-region in the lower mantle are in good agreement with the theoretical data obtained by the model of Feoktistov's uranium-plutonium georeactor with thermal power of 30 TW [17]. Figure 2 shows the special experimental investigation of geologically produced antineu- trinos with KamLAND [3] and an alternative description of these data by our georeactor model [17].

We need to note that, in spite of the fact that the experimental KamLAND-data are well described within the framework of georeactor model [17] (see Figure 2), some geophysicists still have doubts about the existence of georeactor, and the value of georeactor power (30 TW) arouses a special mistrust. In this connection we would like to pay attention to a strange restriction $(W \leq 6.2 \mathrm{TW})$ on the value of nuclear georeactor thermal power $W$, which, unfortunately, is frequently met in the scientific literature recently $[3,4,35,36]$, and strongly masks and distorts the clear understanding of problem of georeactor existence, which is intricate enough by itself. Below we ground a complete inconsistency of this restriction. One of the conclusions of the KamLAND-colloboration is the upper bound of nuclear georeactor thermal power ( $W \leq$ $6.2 \mathrm{TW}$ at $90 \%$ C.L.), which is a direct consequence of uncertainty of KamLAND experimental data [4]. However, it is necessary to keep firmly in mind that the restriction of $6.2 \mathrm{TW}$ on georeactor power is true only for the specific parameters of mixing, i.e. for $\Delta m_{21}^{2}=7.58$ $\times 10^{-5} \mathrm{eV}^{2}, \tan ^{2} \theta_{12}=0.56$, and takes into account the

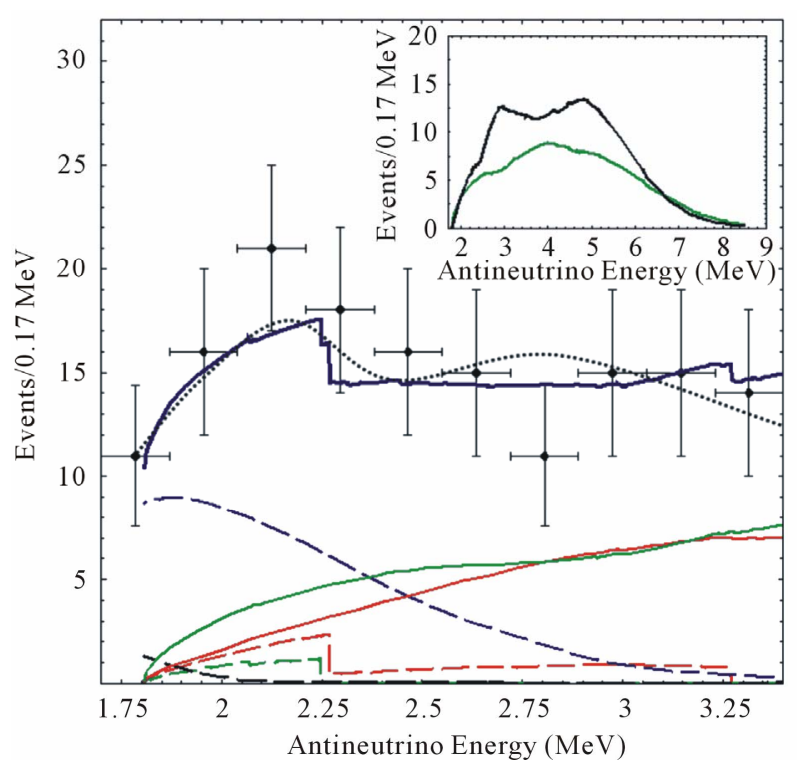

Figure 2. The $\tilde{v}_{e}$ energy spectra in KamLAND [17]. Main panel, experimental points (solid black dots with error bars) together the total expectation obtained in KamLAND experiment (dotted black line) [3] and present paper (thick solid blue line). Also shown are expected neutrino spectrum (solid green line) from Japan's reactors, the expected neutrino spectrum from georeactor 30 TW (red line), the expected signals from ${ }^{238} \mathrm{U}$ (dashed red line) and ${ }^{232} \mathrm{Th}$ (dashed green line) geoneutrinos, ${ }^{13} \mathrm{C}(\alpha, n){ }^{16} \mathrm{O}$ reactions (dashed blue line) and accidentals (dashed black line). Inset: expected spectra obtained in KamLAND experiment (solid black line) [3] and our paper [17] (solid green line) extended to higher energy. 
existence of georeactor within the framework of nonzero hypothesis [4], but absolutely ignores such a nontrivial property of the nuclear georeactor as an uncertainty of georeactor antineutrino spectrum, which in the case of soliton-like nuclear georeactor reaches $\sim 100 \%$. As it will be shown below, the this level of uncertainty within the framework of maximum likelihood function leads (in the minimization of the $\chi^{2}$-function) to a considerable expansion of restriction on the nuclear georeactor heat power $(\sim 30 \mathrm{TW})$ and, accordingly, to the new oscillation parameters $\left(\Delta m_{21}^{2}=2.5 \times 10^{-5} \mathrm{eV}^{2}, \tan ^{2} \theta_{12}=0.437\right)$ for reactor antineutrino.

Another widespread error is related to determination of the Earth geothermal power $W_{\text {Earth. }}$ It is known that there are two estimations of $W_{\text {Earth, i.e., }}$ 33 \pm 1 TW [37] and $\sim 44 \pm 1 \mathrm{TW}$ [38]. We are not going to participate in the discussion among the authors of these estimations concerning the necessity of taking into account the hydrothermal circulation. We would like only to emphasize that these estimations are $1.7-2.3$ times greater than the radiogenic heat contribution (from the decay ${ }^{238} \mathrm{U},{ }^{232} \mathrm{Th}$ and ${ }^{40} \mathrm{~K}$ in the mantle and crust), which is 19.5 TW [17]. D.L. Anderson [39] refers to this difference as "the missing heat source problem" and summarizes the situation in the following words: "Global heat flow estimates range from 30 to $44 \mathrm{TW} \ldots$ Estimates of the radiogenic contribution (from the decay of $\mathrm{U}$, Th and $\mathrm{K}$ in the mantle), based on cosmochemical considerations, vary from 19 to 31 TW. Thus, there is either a good balance between current input and output $\cdots$ or there is a serious missing heat source problem, up to a deficit of $25 \mathrm{TW} \ldots$... Because of this missing heat, some researchers think that, if a reactor exists, its thermal power must make up the existent deficit of geothermal energy. It is correct when it comes to a thermal power of reactor which operated in the remote past, but which does not operate today. The difference between the heat generated now by a reactor in the Earth interior and the experimentally observed geothermal heat $[37,38]$ is very significant due to the high thermal inertia of the Earth. In other words, it is necessary to take into account that the heat generated in the Earth interior is not instantly transferred to the surface, but delays (due to a low heat conductivity) in a time of thermal relaxation of the Earth $\left(\tau_{E} \approx 10^{9}\right.$ years) $[40,41]$. From here it follows that it is impossible to sum up the heat flows which have different spatial and temporal origin.

\section{The Nonstationary Soliton-Like Nuclear Georeactor and KamLAND Antineutrino Spectrum (Experiments over the Period of 2002-2007)}

Now we consider the use of idea of soliton-like nuclear georeactor to describe the KamLAND experimental antineutrino spectra over the period of 2002-2007 [4]. For this purpose let us estimate an uncertainty of nuclear georeactor thermal power and an uncertainty of georeactor antineutrino spectrum, respectively. Note that, generally speaking, such an uranium-plutonium georeactor can consist of a few tens or hundreds of reactors (with the total thermal power of $30 \mathrm{TW}$ ), which represents the individual burning "rivers" and "lakes" of an inhomogeneous actinide shell located in the valleys of rough surface of the Earth's solid core [17]. In the general case, the fission rate of ${ }^{239} \mathrm{Pu}$ nuclei for the uranium-plutonium cycle (1) in the one-group approximation can be written down in the form

$$
\lambda_{\mathrm{Pu}}=\varphi \sigma_{f} n_{\mathrm{Pu}} V
$$

where $\varphi=v n$ is the neutron-flux density; $v$ is the neutron velocity; $n$ is the neutron concentration; $\sigma_{f}$ is the fission cross-section for ${ }^{239} \mathrm{Pu}$; $n_{\mathrm{Pu}}$ is the ${ }^{239} \mathrm{Pu}$ concentration; $V$ is the volume of burning area.

It is easy to see that due to the random character of critical and equilibrium concentrations of plutonium in an actinoid shell and also a stochastic geometry of the "rivers" and "lakes" of actinoid medium, the relative variations of neutron flux density $\varphi$, the plutonium concentration $n$ and the volume of burning areas can run up to $50 \%$ and more. Then ignoring the possible variations of fission cross-section for plutonium, we can write down the following relation for the relative variation of fission rate:

$$
\begin{aligned}
& \frac{\Delta \lambda_{\mathrm{Pu}}}{\lambda_{\mathrm{Pu}}}=\left[\left(\frac{\Delta \varphi}{\varphi}\right)^{2}+\left(\frac{\Delta n_{\mathrm{Pu}}}{n_{\mathrm{Pu}}}\right)^{2}+\left(\frac{\Delta V}{V}\right)^{2}\right]^{1 / 2} \geq 0.87, \\
& \frac{\Delta \sigma}{\sigma} \ll 1
\end{aligned}
$$

On the other hand, it is obvious that a kinetics of georeactor, which operates on the boundary of the liquid and solid phases of the Earth core at the temperature of 5000 - $6000 \mathrm{~K}$ and a pressure of a few hundreds of thousands atmospheres, must necessarily take into account a heat transfer kinetics. This is caused by the fact that under such thermodynamics conditions between these kinetics non-trivial feed-backs can arise, which will significantly change the "traditional" kinetics of neutrons and nuclear reactions. It should be noted that it seems to be the first time that such a problem is being solved within the framework of reactor physics. We have obtained the dependence of fission cross-section $\left\langle\sigma_{f}\right\rangle$ for the ${ }_{94}^{239} \mathrm{Pu}$ nuclei averaged over the neutron spectrum on the nuclear fuel temperature $T$ by the computational experiment with an allowance for the moderation of neutrons and neutron resonance absorption (Figure 3). 


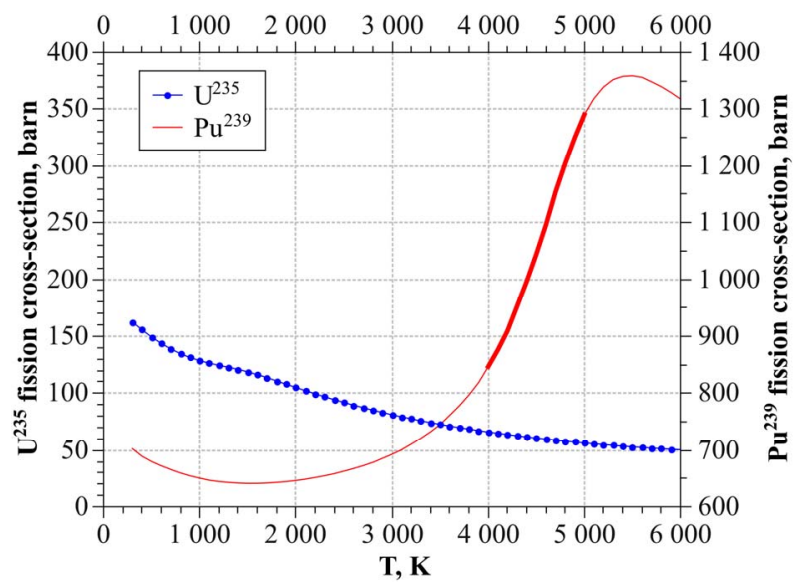

Figure 3. Dependence of ${ }^{239} \mathrm{Pu}$ fission cross-section averaged over the neutron spectrum on fuel medium temperature for limiting energy ( $3 \mathrm{kT})$ of the Fermi and Maxwell spectra. The similar dependence for the ${ }^{235} \mathrm{U}$ fission cross-section is shown for comparison.

This dependence has a form of a power function ${ }^{1}$ in the 4000 to $6000 \mathrm{~K}$ range (see Figure 3):

$$
\left\langle\sigma_{f}^{\mathrm{Pu}}\right\rangle \sim T^{\alpha}, \text { where } \alpha \geq 2 .
$$

From Figure 3 follows that the weak variations of temperature in the 4000 to $6000 \mathrm{~K}$ range can cause the strong variations of fission cross-section $\left\langle\sigma_{f}\right\rangle$ for ${ }^{239} \mathrm{Pu}$, which can run up to $100 \%$ and more. The variations of fission cross-section $\left\langle\sigma_{f}\right\rangle$ for ${ }^{239} \mathrm{Pu}$ will, in their turn, cause the variations of neutron flux density $\varphi$ and neutron concentration $n$. It means that an expression for the fission rate of ${ }^{239} \mathrm{Pu}$ in uranium-plutonium cycle (1), which is analogous to (4), will be more complicated in the multigroup approximation.

However, in spite of the difficulties with analytical determination of the plutonium fission rate variation, it is possible to show (without loss of generality) the lower estimation of relative variation in the case of multigroup approximation

$$
\frac{\Delta \lambda_{\mathrm{Pu}}}{\lambda_{\mathrm{Pu}}} \sim \frac{\Delta \sigma_{f}^{\mathrm{Pu}}}{\sigma_{f}^{\mathrm{Pu}}} \geq 1 .
$$

Now let us show the uncertainty of georeactor antineutrino spectrum with oscillations due to the relative error of plutonium fission rate (5). For this purpose we write down the theoretical form of measured total energy spectrum $\mathrm{d} n_{i} / \mathrm{d} E \equiv n_{i}(E)$ in the $i^{\text {th }}$ energetic bin

$$
n_{i}(E)=m_{\lambda} v_{i}(E) \text {, }
$$

\footnotetext{
${ }^{1}$ It is interesting, that such a behavior of cross-section on the medium temperature is appropriate for the fission cross-section and capture cross-section of ${ }^{239} \mathrm{Pu}$ and absolutely is not appropriate for similar cross-sections of the ${ }^{235} \mathrm{U}$ nuclei, which have the classical dependence of $1 / v$. type.
}

where

$$
\begin{aligned}
& m_{\lambda}=\lambda_{\mathrm{Pu}} \Delta t, \\
& v_{i}(E)=\frac{\varepsilon_{i} N_{p}}{4 \pi L^{2}} \sum_{j, i} \alpha_{i} \rho_{j i}(E) \sigma_{v p}(E) p(E, L), \\
& p(E, l)=1-\sin ^{2}\left(2 \theta_{21}\right) \sin ^{2}\left(\frac{\pi l}{L}\right), \\
& \text { where } L(E)=\frac{2.48 E\left[\mathrm{MeV}^{2}\right]}{\Delta m_{12}^{2}\left[\mathrm{eV}^{2}\right]}[m] .
\end{aligned}
$$

Here $m_{\lambda}$ is the total number of fissions during the exposure time $\Delta t$ determined by the fission rate $\lambda_{\mathrm{Pu}} ; v_{i}(E)$ is the average number of detected antineutrino per fission in the $i^{\text {th }}$ energetic bin; $\varepsilon$ is the detection efficiency for positrons in the inverse $\beta$-decay reaction; $N_{p}$ is the number of protons in the detector sensitive volume; $\Delta t$ is the exposure time; $p(E, L)$ is the neutrino oscillation probability at the appropriate parameters of mixing and energy $E$ at a distance of $l$ from the reactor; $L$ is the oscillation length; $\theta_{21}$ is the mixing angle; $\Delta m_{12}^{2} \equiv\left|m_{2}^{2}-m_{1}^{2}\right|$ is the mass squared difference; $\left(1 / 4 \pi L^{2}\right)$ is the effective solid angle; $\sigma_{v p}$ is the antineutrino-proton interaction cross-section of inverse $\beta$-decay reaction with the corresponding radiation corrections; $\Sigma \alpha_{i} \rho_{i}(E)$ is the nuclear fuel antineutrino energy spectrum in the $i^{\text {th }}$ energetic bin, $\mathrm{MeV} /$ fission; $\alpha_{i}$ is the fraction of $i^{\text {th }}$ isotope.

Here it should be noted that, in general, normalized antineutrino energy spectra corresponding to the different values of reactor heat power may be considered as selfsimilar. This fact simplifies the further analysis considerably. At the same time, a self-similarity takes place only for equilibrium neutrino spectra [42,43], which are typical for stationary processes in reactor core. And vice versa, when processes in the reactor core are nonstationary, a self-similarity of equilibrium neutrino spectra is violated. It means that if, for example, the variations of neutron energy spectrum (and therefore the variations of mass yields induced by the fission of ${ }^{239} \mathrm{Pu}$ ) in the reactor core are considerable, the shapes of corresponding neutrino spectra are not self-similar. Therefore, the calculated ("stationary", i.e. equilibrium) spectra and corresponding experimental (nonstationary) neutrino spectra may differ up to $10 \%-15 \%$ and higher $[42,43]$. The nonequilibrium effect of neutrino spectra will be considered in detail in Section 6.

Obviously, due to the stochastic change of the nonstationary nuclear georeactor power as a consequence of the ${ }^{239} \mathrm{Pu}$ fission cross-section $\left\langle\sigma_{f}\right\rangle$ and the georeactor neutrino spectrum shape (9) variations, the relative uncertainty of georeactor antineutrino spectrum $n_{i}^{g r n}(E)$ with oscillations in the $i^{\text {th }}$ energy bin (with an allowance for Equations (6)-(9)) looks like 


$$
\frac{\Delta n_{i}^{g r n}}{n_{i}^{g r n}} \simeq\left[\left(\frac{\Delta \lambda_{\mathrm{Pu}}}{\lambda_{\mathrm{Pu}}}\right)^{2}+\left(\frac{\Delta \rho_{i}}{\rho_{i}}\right)^{2}\right]^{1 / 2} \geq 1,
$$

where $\left(\Delta \rho_{i} / \rho_{i}\right) \geq 10 \%$ is the relative uncertainty due to nonstationarity of georeactor neutrino spectrum shape.

Therefore the lower estimation of uncertainty of total antineutrino spectrum with oscillations with an allowance for Equation (11) and the contribution of uncertainty $(4.14 \%)$ of antineutrino spectrum $n_{i}^{J a p}(E)$ from the Japanese reactors [4] takes on the form

$$
\Delta n_{i} \geq\left[\left(0.0414 n_{i}^{J a p}\right)^{2}+\left(n_{i}^{g r n}\right)^{2}\right]^{1 / 2} .
$$

Note that this uncertainty is the one shown in Figure 4 as a violet band.

Now we are ready to use our model of uranium-Plutonium georeactor [17] for the alternative description of the data produced in new KamLAND experiment [4]. Obviously the standard methods of obtaining consistent estimates (e.g. the maximum-likelihood method) normally used for the determination of the oscillation parameters $\left(\Delta m_{12}^{2}, \sin ^{2} 2 \theta_{12}\right)$ [1-5] must take into account one more reactor, or, more specifically, take into account the antineutrino spectrum of georeactor with the power of 30 TW which is located at a depth of $L \sim 5.2 \times 10^{6} \mathrm{~m}$. However, following [17], we propose here a simple estimating approach. The results of its application show that the hypothesis of the georeactor existence on the boundary of liquid and solid phases of the Earth core does not contradict the experimental data.

So, we proceed as in [17]; if CPT-invariance is assumed, the probabilities of the $v_{e} \rightarrow v_{e}$ and $\tilde{v}_{e} \rightarrow \tilde{v}_{e}$ oscillations should be equal at the same values $L / E$. On the other hand, it is known that the variations of $\Delta m^{2}$ dominate over the more stable small variations of angle $\theta$ at the spectral distortion (oscillations) of "solar" neutrino spectrum. Therefore we can assume (on the grounds of CPT-theorem) that the angle which is determined by the experimental "solar" equality $\tan ^{2} \theta_{12}=0.447$ [44] may be used as the reference angle of mixing in the KamLAND-experiment.

Finally, following the computational ideology of [17], we give the results of verification of the optimal oscillation parameters $\left(\Delta m_{21}^{2}=2.5 \times 10^{-5} \mathrm{eV}^{2}, \tan ^{2} \theta_{12}=0.437\right)$ by comparing the theoretical (which takes into account the georeactor operation) and experimental spectra of reactor antineutrino based on the KamLAND data over the period of 2002-2007 (Figure 4). We compare also in Figure 5 the $\chi^{2}$-profiles for our georeactor hypothesis and KamLAND nonzero hypothesis, which does not take into account an uncertainty of reactor antineutrino spectrum (see Section 6).

In spite of low statistics of neutrino events $(\leq 150$

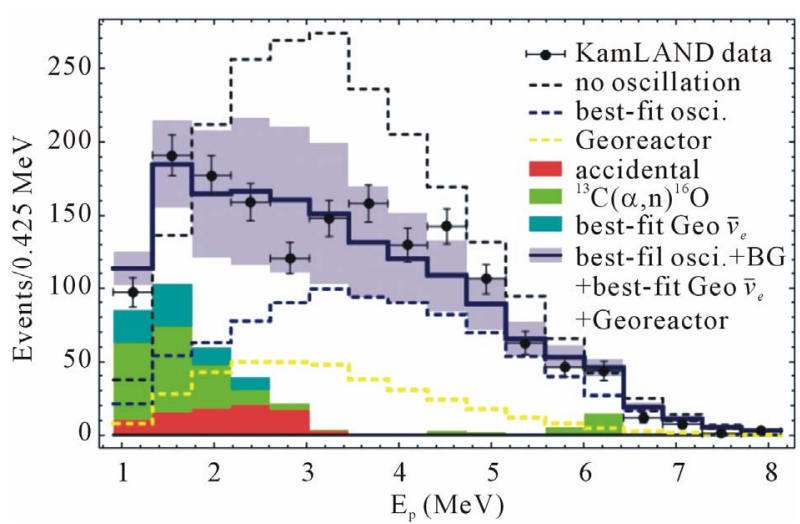

Figure 4. Prompt event energy spectrum of $\tilde{v}_{e}$ candidate events (2002-2007). The shaded background and geoneutrino histograms are cumulative. Statistical uncertainties are shown for the data; the violet band on the blue histogram indicates the event rate systematic uncertainty. The georeactor power is 19.5 TW. The georeactor is at a distance of $5098 \mathrm{~km}$ from the KamLAND-detector (see explanation in the text and Table 1).

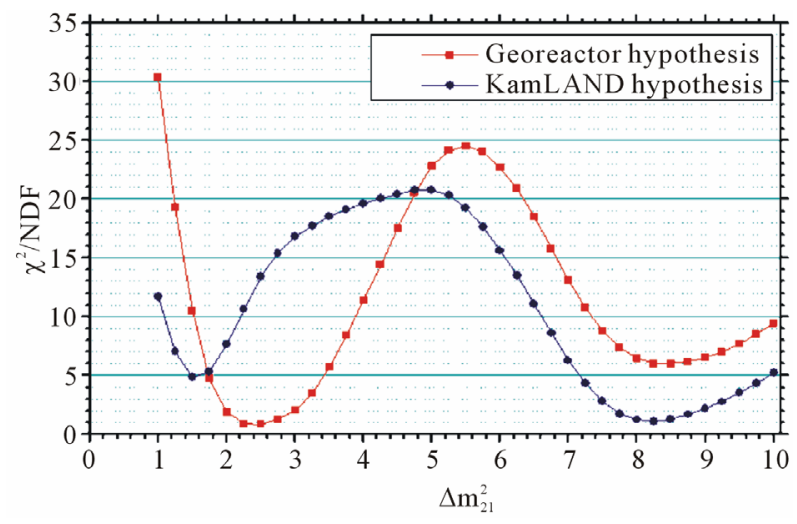

Figure 5. Dependences of $\chi^{2} / \mathrm{NDF}$ on the mass squared difference $\Delta m_{21}^{2}$ corresponding to KamLAND-hypothesis without georeactor (blue line, $\tan ^{2} \theta_{12}=0.56$ [4]) and our georeactor hypothesis (red line, $\tan ^{2} \theta_{12}=0.437$ ).

events/bin), the theoretical reactor antineutrino spectrum (which takes into account a soliton-like nuclear georeactor with the power of 19.5 TW) describes with an acceptable accuracy the experimental KamLAND-data (Figure 4) [4]. Below we pay attention to some important moments.

\subsection{Peculiarities of the Low Antineutrino Statistics Accounting}

It is obvious, that a low antineutrino statistics is the reason of high inhomogeneity of filling event density of an antineutrino spectrum (which is continuous by its nature). This inhomogeneity intensifies due to energy discretization of spectrum ( $0.425 \mathrm{MeV}$ in KamLAND-experiment). As a result, the formal procedure of events integration 
within one energy bin $(\Delta E=0.425 \mathrm{MeV})$ can generate substantial deviations of the number of events (per bin) from its true average value. To observe this effect it would be necessary to decrease the width of energy bin considerably or, that is the same, to decrease the width of detector energy window. However, since it is impossible, we will attempt to show this effect in the following way.

As follows from Figure 4, the significant disagreement between the experimental and theoretical antineutrino spectra is observed for the 5, 7 and 9 bins. Therefore, if the apparent condition

$$
P(l)=\left(1-p\left(E_{5}, l\right)\right) p\left(E_{7}, l\right) p\left(E_{9}, l\right)=\max ,
$$

is imposed on oscillations of nuclear georeactor antineutrino spectrum, then by Equation (10) and the average energies of bins $E_{5}=2.8 \mathrm{MeV}, E_{7}=3.7 \mathrm{MeV}$ and $E_{9}=$ $4.5 \mathrm{MeV}$ (see Figure 4) we can obtain a series of the values $l$ for distances from KamLAND-detector to the supposed location of georeactor on the surface of Earth solid core (Figure 6).

$$
l=5365,5968,6400,6830,7410 \mathrm{~km} .
$$

Now let us return to the problem of low antineutrino statistics. Fulfillment of condition (13) for given distances (14) makes it possible to recalculate a georeactor antineutrino spectrum (Figure 4) for these distances by Equations (8)-(10). Proceeding from a low antineutrino statistics (in energy bins $E_{5}=2.8 \mathrm{MeV}, E_{7}=3.7 \mathrm{MeV}$ and $E_{9}=4.5 \mathrm{MeV}$ ), the following variants of the location of a georeactor on the Earth solid core surface are most acceptable: 1) a georeactor with the thermal power of $30.7 \mathrm{TW}$ at $6400 \mathrm{~km}$ distance from KamLAND detector (Figure 7(a)); 2) a georeactor with the thermal power of $34.7 \mathrm{TW}$ at $6830 \mathrm{~km}$ distance from KamLAND detector (Figure 7(b)); georeactors of equal thermal power but

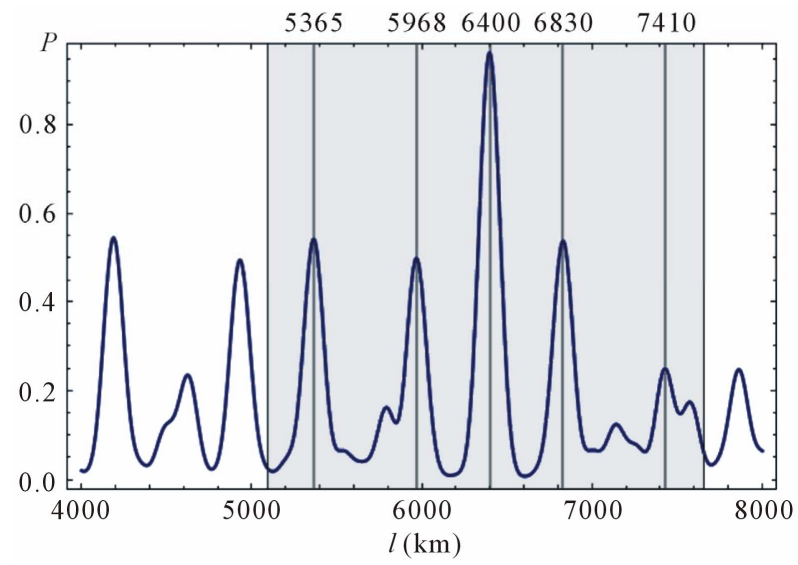

Figure 6. The spatial frequency distribution $P(l)$ of oscillating georeactor antineutrinos with energies $E_{5}=2.8 \mathrm{MeV}, E_{7}$ $=3.7 \mathrm{MeV}$ и $E_{9}=4.5 \mathrm{MeV}$. Shaded area corresponds to the continuous series of distances $l$ from the KamLAND-detector to the Earth solid core surface.

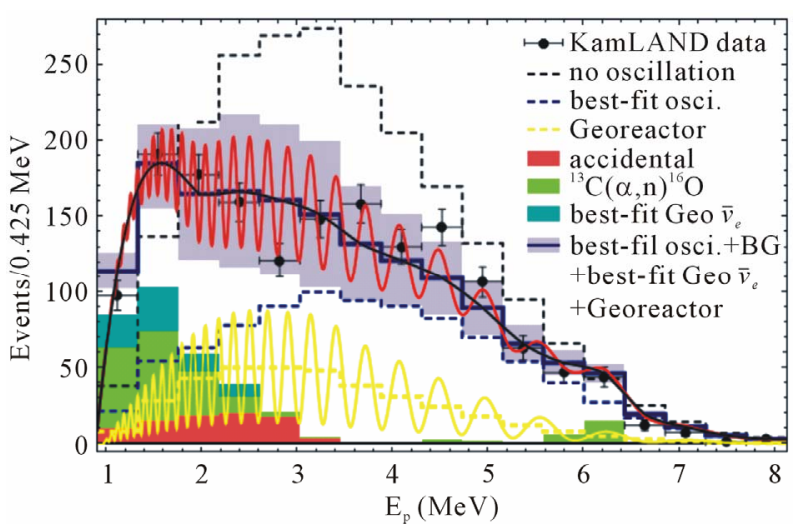

(a)

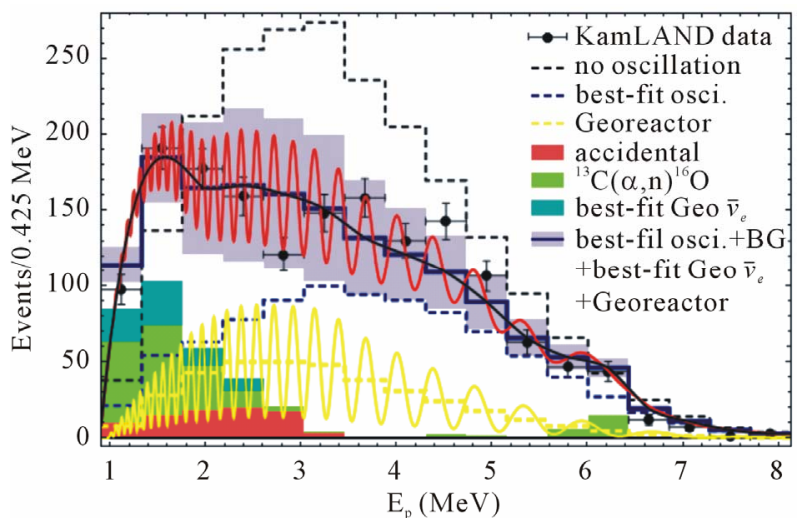

(b)

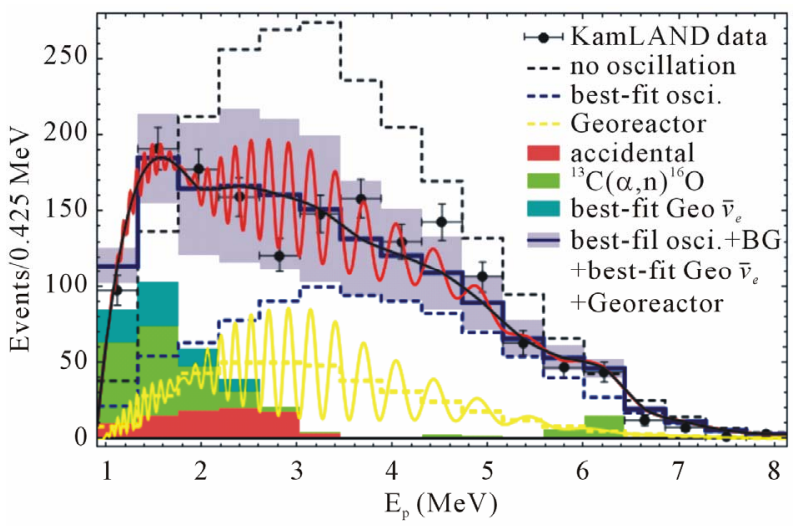

(c)

Figure 7. The theoretical antineutrino spectrum (blue histogram), which takes into account the nuclear georeactor (a) with thermal power of $30.7 \mathrm{TW}$ (yellow histogram) situated at a distance of $6400 \mathrm{~km}$ from the KamLAND-detector, (b) with thermal power of 34.7 TW (yellow histogram) situated at a distance of $6830 \mathrm{~km}$ from the KamLAND-detector, (c) with general thermal power 32.6 TW (yellow histogram) situated simultaneously at the distances of 6400 and 6830 $\mathrm{km}$ from the KamLAND-detector. In all figures one can see how discrete antineutrino spectra of KamLAND-experiment (blue histogram) and georeactor (yellow histogram) mask the low statistics effect in corresponding continuous antineutrino spectra (red and yellow oscillations). 
with the total power of $32.6 \mathrm{TW}$ at 6400 and $6830 \mathrm{~km}$ distance from KamLAND detector (Figure 7(c)).

These results force us to recalculate the georeactor thermal power obtained for KamLAND data over the period of 2002-2004 [17]. The parameters of nuclear georeactors obtained by two calculation methods of antineutrino spectra over the periods of 2002-2004 and 20022007 are collected in Table $\mathbf{1 .}$

The considered peculiarities of low antineutrino statistics make it possible not only to determine the possible distances from KamLAND detector to supposed nuclear georeactor on the Earth solid core surface (Figure 6), but to construct the map of located on the Earth surface lines radially conjugate to lines-circumferences formed by the bases of cones with a vertex in KamLAND and generating sides with lengths equal to the corresponding distances from KamLAND detector to the Earth solid core surface (Figure 8).

\subsection{Non-Stationary Nature of Soliton-Like Nuclear Georeactor}

Analyzing Table 1, we can see that within the framework of modified method for calculation of antineutrino spectrum (Figure 7), the average thermal power of georeactor changes from $\sim 50 \mathrm{TW}$ (during the exposure of 749.1 days in 2005 [3], Figure 2) to $\sim 30 \mathrm{TW}$ (at total exposure of 1890.25 days in 2008 [4]). There is no doubt

Table 1. Nuclear georeactor thermal power depending on a distance to detector and exposure time in the KamLAND and Borexino experiments.

\begin{tabular}{|c|c|c|c|c|c|c|}
\hline Period & $2002-2004$ & $2002-2007$ & $2005-2007$ & 2002-2009 & 2008-2009 & 2008-2009 \\
\hline Experiment & & & KamLAND & & & Borexino \\
\hline Exposure, days & 749.10 & 1890.25 & 1141.15 & 2135 & 244.75 & 537.20 \\
\hline Distance, $\mathrm{km}$ & \multicolumn{6}{|c|}{ Nuclear Georeactor Power, TW } \\
\hline 5098 & 30.0 & 19.5 & 12.6 & 17.3 & 4.7 & - \\
\hline 6400 & 47.3 & 30.7 & 19.8 & 28.0 & 7.1 & - \\
\hline 6830 & 53.4 & 34.7 & 22.4 & 31.6 & 7.7 & - \\
\hline $6400+6830$ & 50.2 & 32.6 & 21.1 & 29.7 & 7.3 & - \\
\hline 6711 & - & - & - & - & - & 5.0 \\
\hline
\end{tabular}

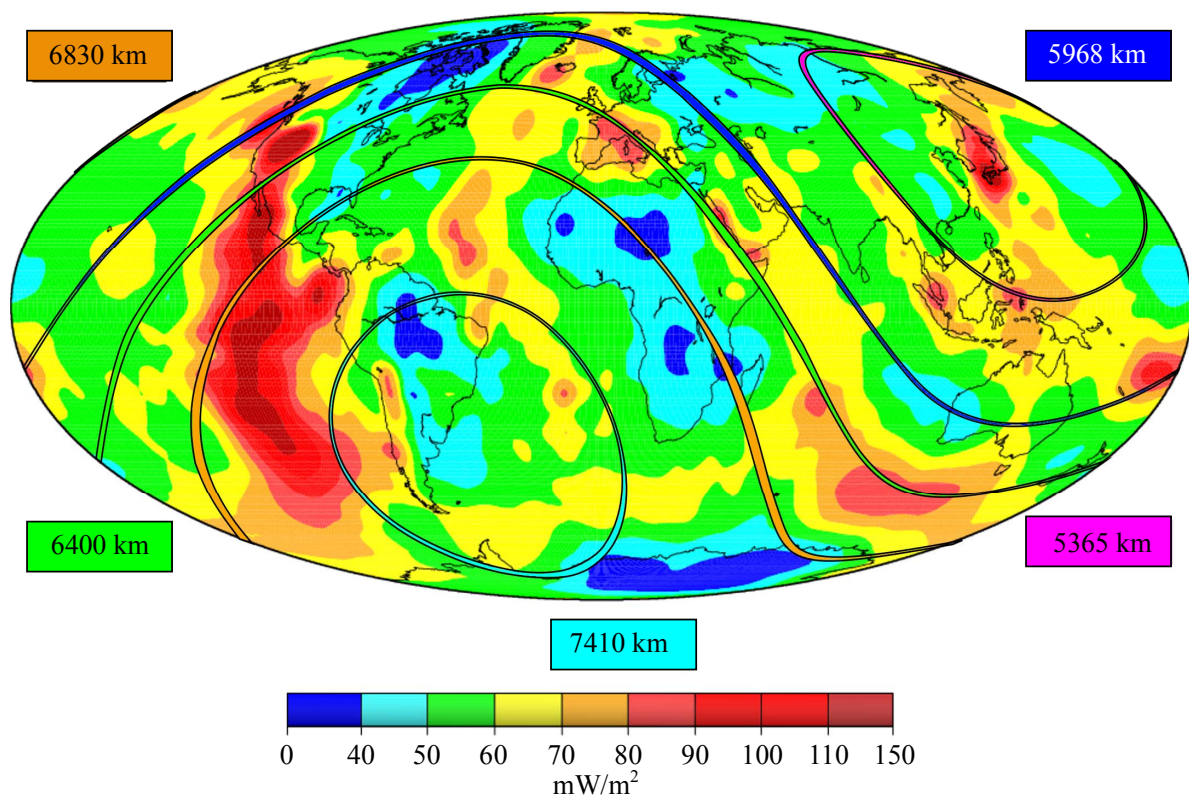

Figure 8. Distribution of geothermal power density on the Earth [45] superposed with the conjugated "pseudoreactor" circumferences, which are generated by "georeactor" circumferences located on the perimeters of the bases of cones with a vertex in KamLAND $\left(36.43^{\circ} \mathrm{N}\right.$ and $\left.137.31^{\circ} \mathrm{E}\right)$ and generating sides with lengths from KamLAND-detector to the Earth solid core surface equal to 7410 (sky blue), 6830 (orange), 6400 (green), 5968 (blue) and 5365 (pink) km. 
that it is the reflection of a non-stationary nature of georeactor. Taking into account that total exposure (1890.25 days) is a sum of two consecutive exposures (749.1 and 1141.15 days, respectively), in fact the change in the average thermal power of georeactor is even more, i.e. from $\sim 50 \mathrm{TW}$ over the first exposure to $\sim 20 \mathrm{TW}$ over the second consecutive exposure (see Table 1).

From the physical standpoint, the decrease of georeactor thermal power (during the two successive exposures) almost by a factor of two means that the variances of fission cross-section $\left\langle\sigma_{f}\right\rangle$ for the ${ }_{94}^{239} \mathrm{Pu}$ nuclei during total exposure over the period of 2002-2007 also changed in two times, or more exactly, went almost $100 \%$ down. It means that the variance of fission cross-section $\left\langle\sigma_{f}\right\rangle$ for the ${ }_{94}^{239} \mathrm{Pu}$ nuclei reaches practically $100 \%$ and this is in good agreement with our estimation of variance of fission rate (7).

At the same time, we asserted before that the main cause of change of fission cross-section $\left\langle\sigma_{f}\right\rangle$ for the ${ }_{94}^{239} \mathrm{Pu}$ nuclei under extreme thermodynamics conditions are the temperature variations of fuel medium. Therefore, there is a natural question as to what (except the georeactor) is the reason of the fuel temperature variations, or more exactly, what is the physical nature of independent source of the fuel temperature variations, which in the end plays the role of external modulator of nuclear georeactor thermal power. The answer to this very important question related to finding out the physical reasons of nonstationary nature of soliton-like nuclear georeactor will be given in the second part of the present paper [46].

Briefly summarizing the results of this section, we can say that in spite of the low statistics of neutrino events ( $\leq 150$ events/bin), the theoretical reactor antineutrino spectrum (which takes into account the soliton-like nuclear georeactor with the power of $30 \mathrm{TW}$ ) describes with acceptable accuracy the experimental KamLANDdata [4] (see Figures 4 and 7). Here we pay attention to some important moments. First, the average georeactor heat power is changed from $\sim 50 \mathrm{TW}$ at the exposure time of 749.1 days in 2005 [3] (Figure 2) to $\sim 30 \mathrm{TW}$ at total exposure of 1890 days in 2008 [4] (Figure 3). This reflects the nonstationary nature of the georeactor.

\section{The Borexino and KamLAND Experiments and Triangulation of Soliton-Like Nuclear Georeactors Location}

As is generally known, the first stage of Borexino experiment (Laboratory Nationali del Gran Sasso, Italy) [47] was recently completed, ideology of neutrino measurements in which is practically analogical to that of the neutrino measurements in KamLAND experiment. It means that the joint use of the Borexino and KamLAND data opens up non-trivial possibility for the solving of very important problem of spatial identification of nuclear georeactor location on the Earth solid core surface, or in other words, the triangulation of the soliton-like nuclear georeactor location on the boundary of the liquid and solid phases of the Earth core.

\subsection{Borexino Antineutrino Spectrum (Exposure over the Period of 2008-2009)}

We give here the alternative analysis of the Borexino data collected between December 2007 and December 2009, corresponding to 537.2 days of live time [46]. The fiducial exposure after all selection cuts is 252.6 ton·yr. The determination of the expected neutrino signal from reactors, which, as usual, was calculated by Equation (8), required the collection of the detailed information on the time profiles of power and nuclear fuel composition for nearby reactors. In Equation (8) the main contribution comes from 194 reactors in Europe, while other 245 reactors around the world contribute only $2.5 \%$ of the total reactor signal. Information on the nominal thermal power and monthly load factor for each European reactor originate from IAEA and EDF [47].

It is important to note that to describe the antineutrino spectra in the Borexino experiment, the parameters of mixing $\left(\Delta m_{21}^{2}=7.65 \times 10^{-5} \mathrm{eV}^{2}, \sin ^{2} \theta_{12}=0.304\right.$ [48]) based on the global three-flavour analysis of solar (SNO) and reactor (KamLAND) experimental data were used. At the same time, for the alternative description of the Borexino data (Figure 9) we use the parameters of mixing $\Delta m_{21}^{2}=2.5 \times 10^{-5} \mathrm{eV}^{2}, \tan ^{2} \theta_{12}=0.437$, or in other words, $\sin ^{2} \theta_{12}=0.304$ obtained by our model, which takes into account the existence of natural nuclear reactor on the boundary of the liquid and solid phases of the Earth core [17].

It is obvious, that in spite of very low statistics of neutrino (reactor) events ( $1-2$ events/bin), the theoretical reactor antineutrino spectrum (which takes into account a soliton-like nuclear georeactor with the power of $5 \mathrm{TW}$ ) describes with an acceptable accuracy the experimental Borexino data (Figure 9) [47]. Note that the lower estimation of uncertainty of total antineutrino spectrum with oscillations (which is headlined in violet colour in Figure 9) was calculated by Equation (12) at the uncertainty of antineutrino spectrum $n_{i}^{\text {Euro }}(E)$ from European reactors equal to $5.38 \%$ [47].

\subsection{Peculiarities of the Low Antineutrino Statistics Accounting}

As follows from Figure 9, the considerable disagreement between the experimental and theoretical antineutrino spectra is observed for 5, 6, 7 and 11 bins. Therefore, if the apparent condition 


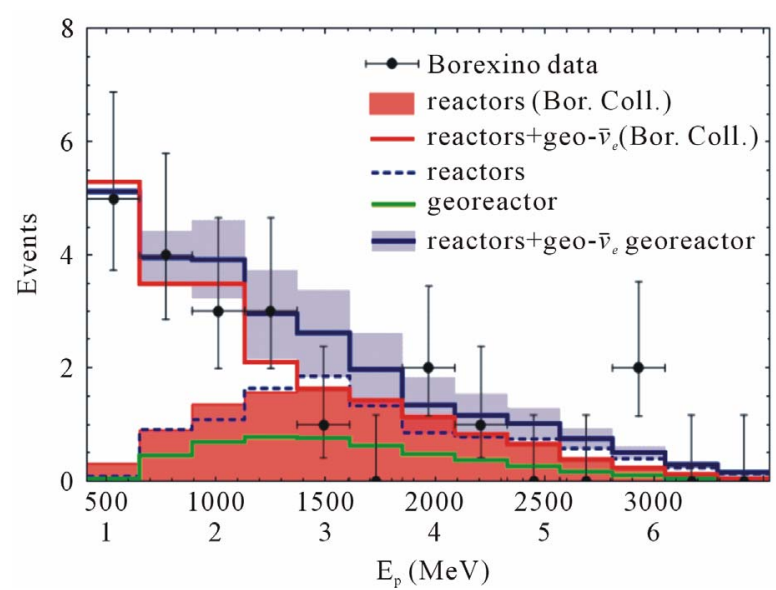

Figure 9. The $\tilde{\boldsymbol{v}}_{e}$ energy spectra in Borexino [47]. Experimental points (solid black dots with error bars) together with the total expectation obtained in Borexino experiment (red solid histogram) [3] and in the present paper (blue solid histogram). Also shown are the expected neutrino spectrum from European reactors calculated using our parameters of mixing (blue dashed histogram) and obtained in Borexino-experiment (red shaded area). The expected neutrino spectrum from the georeactor of $5 \mathrm{TW}$ located at a distance of $6711 \mathrm{~km}$ from the Borexino-detector (green line) is also shown (see explanation in the text).

$$
\begin{aligned}
P(l)= & \left(1-p\left(E_{5}, l\right)\right)\left(1-p\left(E_{6}, l\right)\right) \\
& \times p\left(E_{7}, l\right) p\left(E_{11}, l\right)=\max ,
\end{aligned}
$$

is imposed on the oscillations of nuclear georeactor antineutrino spectrum, then by Equation (15) for $p(E, l)$ and the bin average energies $E_{5}, E_{6}, E_{7}$ and $E_{11}$ (see Figure 9) we can obtain a series of the values $l$ for possible distances from Borexino-detector to the supposed location of georeactor on the surface of Earth solid core (Figure 10).

$$
l=5310,5400,6310,6711,7128,7490 \mathrm{~km} .
$$

Returning to the problem of low antineutrino statistics, note that the fulfillment of the condition (15) for given distances (16) makes it possible to recalculate by Equations (8)-(10) a georeactor antineutrino spectrum (Figure 9) for these distances. Proceeding from a low antineutrino statistics (in energy bins $E_{5}, E_{6}, E_{7}$ and $E_{11}$ ), the most acceptable location of a georeactor on the Earth solid core surface is at $6711 \mathrm{~km}$ distance from the Borexino detector (Figure 10). It is easy to show that the thermal power of such a nuclear georeactor is $\sim 5 \mathrm{TW}$.

It is necessary to note that the restriction on the nuclear georeactor thermal power obtained within the framework of the Borexino experiment is $\sim 3$ TW [47]. Though this restriction is obtained within the framework of nonzero georeactor hypothesis, it does not take into account the high uncertainty of georeactor antineutrino spectrum. The consequences of neglect of this uncertainty come into a question in Section 6.

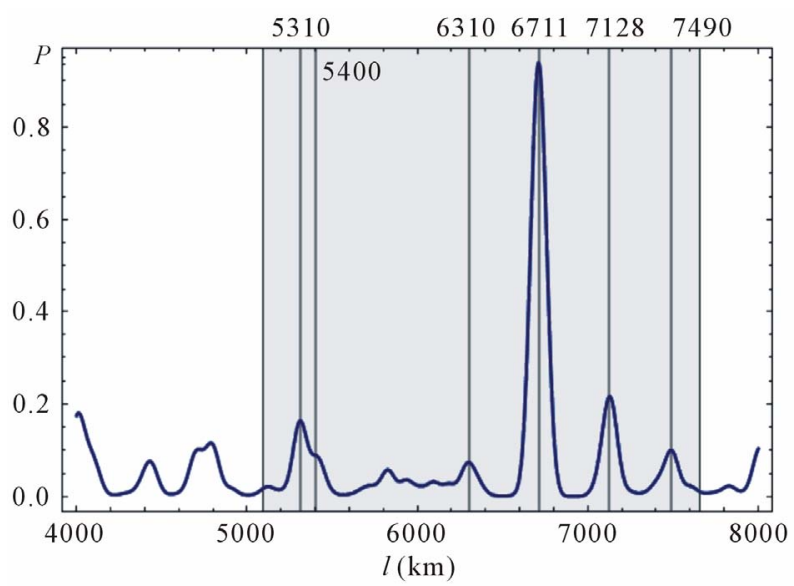

Figure 10. The spatial frequency distribution $P(l)$ of oscillating georeactor antineutrinos with energies corresponding to 5, 6, 7 and 11 bins in Borexino-spectrum in Figure 9. Shaded area corresponds to the continuous series of the distances $l$ from the Borexino-detector to the surface of the Earth solid core.

\subsection{Triangulation of the Locations of Soliton-Like Nuclear Georeactors}

By triangulation of the KamLAND and Borexino data we have constructed the "pseudogeoreactor" coordinate location conjugate to the real location of soliton-like nuclear georeactors on the boundary of the liquid and solid phases of the Earth core (Figure 11).

Analyzing Figures 6 and 7 and also Figure 10 (by which it is possible to determine the most probable distances between the detector and a nuclear georector), we have divided georeactors into two groups-operating reactors and probable low-power reactors (Figure 11).

Naturally, a question is the following: What is the cause of sufficiently high degree of correlation between "conjugate pseudoreactors" and the regions of higher geothermal power of the Earth in Figure 11? Below we consider physical reasons causing such a correlation.

Here it should be recalled, that according to our assumption [17], nuclear georeactors are located in the thin uranium-containing high-density layer (about $2.2 \mathrm{~km}$ ) [33], which is the peculiar physical boundary of the liquid and solid phases of the Earth core. According to the results of seismic tomography [33], this layer has a mosaic structure with typical size of $\sim 200 \mathrm{~km}$. This means that the spatial history of nuclear burning wave, or in other words "burning spot", is completely determined by the area of one patch of a mosaic structure. Since the area on the Earth surface conjugate to the single patch of a mosaic structure has the typical size $\sim 1000 \mathrm{~km}$, this value is the size of the uncertainty domain for the "conjugate burning spot" on the Earth surface.

On the other hand, we know that the time of heat transmission from the "burning spot" to the "conjugate 
burning spot" has the order of $10^{9}$ years. Taking into account the average velocity of nuclear burning wave $(\sim 1$ $\mathrm{m} /$ year), we obtain that for $10^{9}$ years the "burning spot" will cover the total distance of $10^{6} \mathrm{~km}$ on the Earth surface. Since this distance easily goes into the area of one patch of a mosaic structure in the form of a certain fractal-broken curve, the domain of uncertainty of thermal flow (on the Earth surface) coincides with the domain of uncertainty of the "conjugate burning spot". This coincidence is indeed a reason of good correlation between "conjugate burning spots" location and the areas of higher geothermal power in Figure 11. In other words, a map of geothermal power distribution on the Earth is at the same time a rough approximation of the nuclear georeactors that are acting or had been acting in the past with their locations determined in this case with an accuracy up to $1000 \mathrm{~km}$.

It is interesting that if any considerable geothermal heat release is absent in the places, where the nuclear georectors location is supposed (see Figure 11), the large so-called solitary volcanoes (hot spots), e.g. in the Central and South-East Africa (Figure 12), or active volcanoes, e.g. Erebus in Antarctica (Ross Sea coast), Deception (South Scotch Islands) and a recently discovered

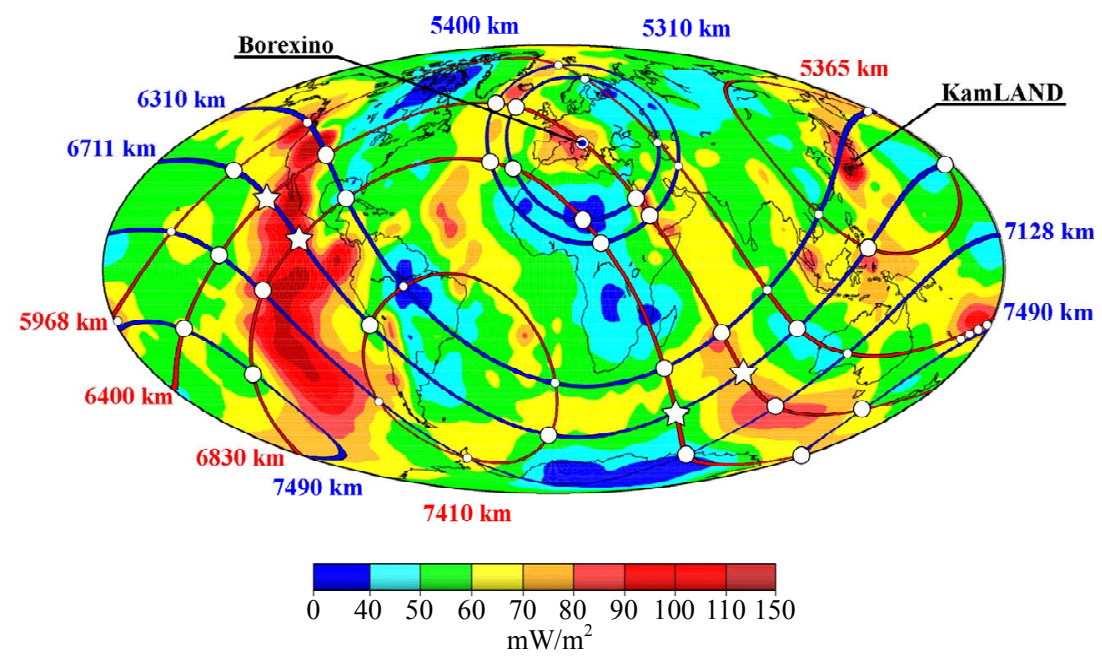

Figure 11. Distribution of geothermal power density on the Earth [45] superposed with the conjugate "pseudogeoreactor" ellipsoidal closed curves, which were built on basis of KamLAND (red lines) and Borexino (blue lines) experimental data. $\left(\zeta^{2}\right)$ - operating nuclear georeactors; $(O)$ and $(\circ)$ - nuclear georeactors with a power (if they are operating) of an order of magnitude and more less than the thermal power of reactors designated by $(\hbar)$.

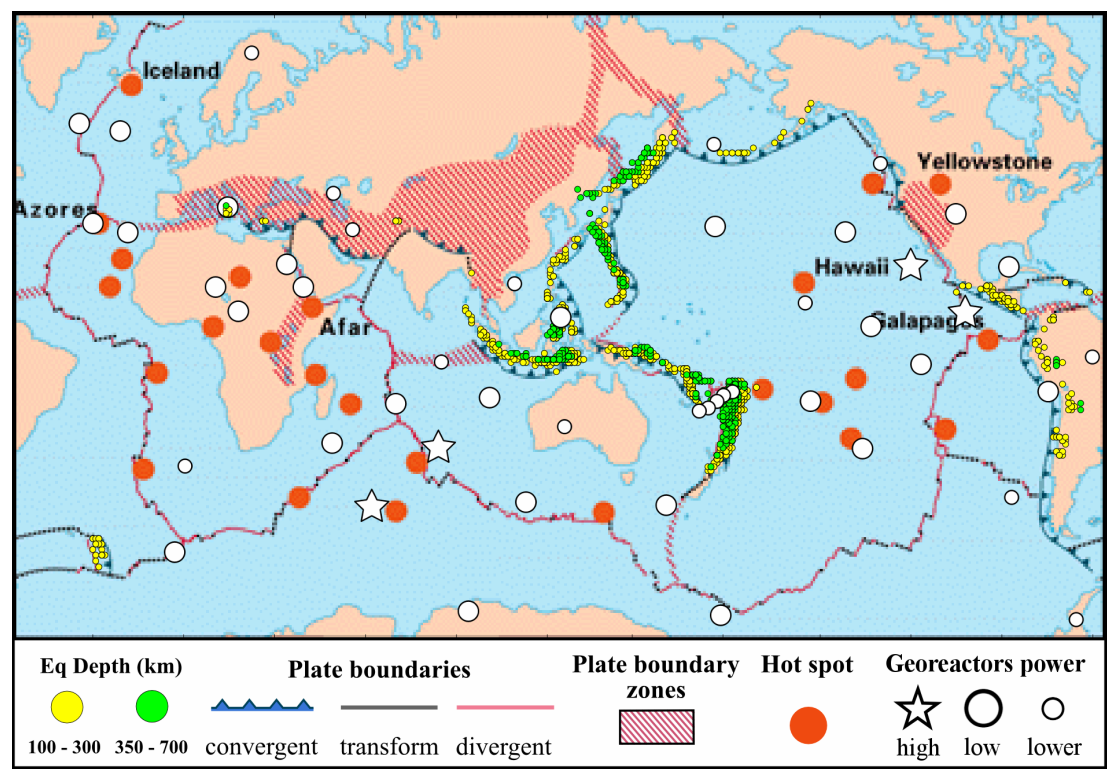

Figure 12. The map of spatial location of "pseudureactors", hot spots and deep-focus earthquakes over the period of 19932006. The map of "pseudureactors" and earthquakes was built on basis of the plate boundaries map [50]. 
nameless volcano (Hudson Mountains in the West Antarctica [49]) are necessarily there.

\section{Non-Stationary Soliton-Like Nuclear Georeactor and New KamLAND Antineutrino Spectrum (Exposure over the Period of 2002-2009)}

At the time this paper was written, another data of KamLAND experiment obtained over the years of 2002-2009 were published [5]. In spite of this, we decided not to change the structure of the paper, but to add the analysis of these data to existing material, because such an analysis is the natural illustration of inner consistency of the considered above georeactor hypothesis.

\section{KamLAND Antineutrino Spectrum}

We give here the alternative description of KamLANDdata [5] collected from March 9, 2002, to November 4, 2009 , corresponding to 2135 days of live time. The number of target protons within the 6.0-m-radius spherical fiducial volume is calculated to be $(5.98 \pm 0.12) \times 10^{31}$ for the combined data set which corresponds to an exposure to electron antineutrino $\tilde{v}_{e}$ of $3.49 \times 10^{32}$ protonyears. The determination of the expected neutrino signal from reactors, which was traditionally calculated by Equation (8), required the collection of the detailed information on the time profiles of power and nuclear fuel composition for nearby reactors. The relative fission yields, averaged over the entire live-time period, for isotopes $\left({ }^{235} \mathrm{U}:{ }^{238} \mathrm{U}:{ }^{239} \mathrm{Pu}:{ }^{241} \mathrm{Pu}\right)$ are $(0.571: 0.078: 0.295$ : 0.065), respectively. In Equation (8) the main contribution comes from 56 Japanese nuclear power reactors, while the contributions from Korean reactors and the remainder of the global nuclear power industry is estimated to be $(3.4 \pm 0.3) \%$ and $(1.0 \pm 0.5) \%$ of the total reactor signal, respectively. Information on the nominal thermal power and monthly load factor for each Japanese reactor originate from consortium of Japanese electric power companies [5].

It is obvious, that the theoretical reactor antineutrino spectrum (which takes into account a soliton-like nuclear georeactor with the power of 29.7 TW (see Table 1)) describes with an acceptable accuracy the new experimental KamLAND-data (Figure 13). Let us note that the lower estimation of uncertainty of total antineutrino spectrum with oscillations is calculated by Equation (12) with an allowance for the contribution of the uncertainty $4.5 \%$ (which corresponds to the variant DS-2 [5]) of total antineutrino spectrum from the Japanese reactors.

It follows from Table 1 that the average thermal power $W_{t}$ of nuclear georeactor sharply decreases in KamLAND experiments corresponding to the exposures over the periods of 2002-2004, 2002-2007 and 2002-2009

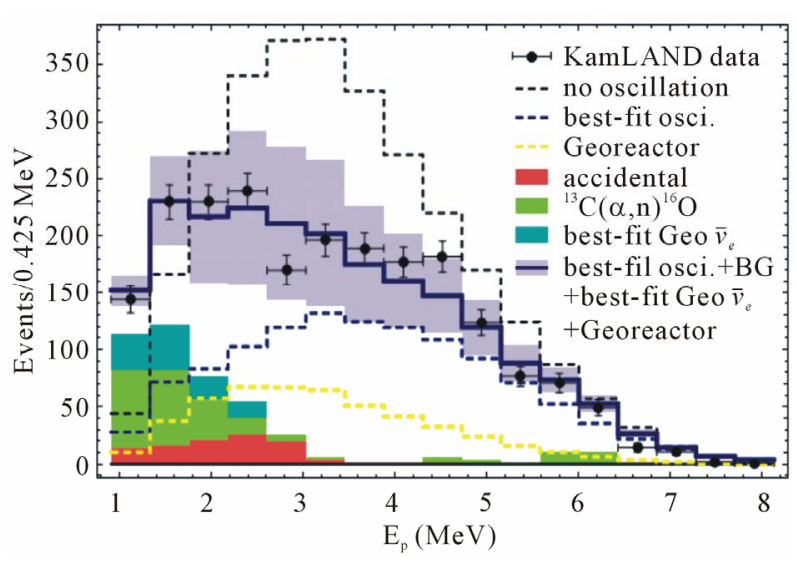

Figure 13. Prompt event energy spectrum of $\tilde{v}_{e}$ candidate events (exposure over the years of 2002-2009). The shaded background and geoneutrino histograms are cumulative. Statistical uncertainties are shown for the data; the violet band on the blue histogram indicates the event rate systematic uncertainty in the framework of georeactor hypothesis. The total georeactor power is $29.7 \mathrm{TW}$. Georeactors are located at a distance of 6400 and $6830 \mathrm{~km}$ from the KamLAND-detector (see explanation in the text and Table 1).

Such a power jump indicates that the nuclear georeactor is strongly nonstationary. It is a very important fact for the correct calculation of reactor geoneutrinos, which in the end are the integral part of KamLAND antineutrino spectrum (within the framework of georeactor hypothesis). To illustrate such a strong nonstationarity we give below the change of the georeactor average thermal power over the period of 2002-2009.

Using the average values of nuclear georeactor thermal power $W_{t}$ reconstructed within the framework of georeactor hypothesis (Table 1), which correspond to exposures over the years of 2002-2004, 2002-2007 and 20022009 , it is possible to determine the values $W_{t}$ corresponding to "latent" exposures over the years of 20052007 and 2008-2009 by obvious expression

$$
\begin{aligned}
& W_{t}=\frac{t_{1}}{t_{1}+t_{2}} W_{t_{1}}+\frac{t_{2}}{t_{1}+t_{2}} W_{t_{2}}, \\
& \text { where } t=t_{1}+t_{2} .
\end{aligned}
$$

The values of nuclear georeactor thermal power $W_{t}$ extended in that way with consideration of exposure over the period of 2002-2004 (see Table 1) make it possible to build the time evolution of the georeactor average thermal power $W$ over the years of 2002-2009 (Figure 14).

Thus, the sharp change of the georeactor average thermal power $W$ during the years of 2002-2009 must be necessarily taken into account in the calculation of the georeactor neutrino spectra, which are the part of the KamLAND antineutrino spectra within the framework of georeactor hypothesis. For that purpose, within the 


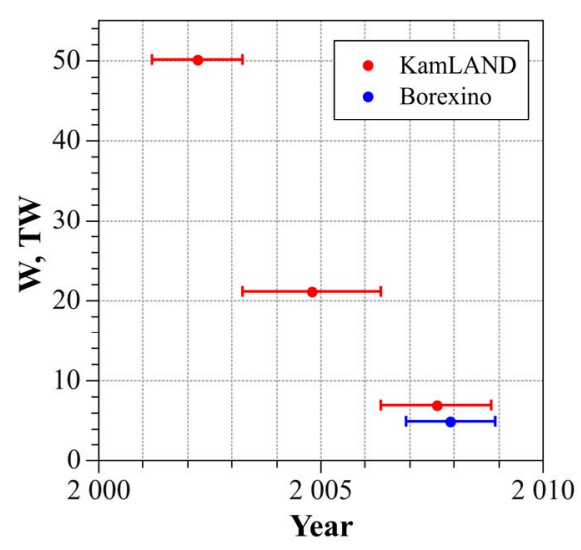

Figure 14. Evolution of reconstructed average thermal power $W$ of nuclear georeactor over the years of 2002-2009.

framework of traditional calculations of effective neutrino spectra of both individual nuclides and their mixture $[42,43]$, it is necessary to take into account the high degree of nonequilibrium of neutrino spectra due to strong nonstationarity of nuclear georeactor power $[42,43]$.

It is very important to note, that in the real experiment exactly the high degree of nonequilibrium of neutrino spectra due to the "latent" nonstationarity of radiation source can become the reason of sharp change of the expected "equilibrium" shape of resulting neutrino spectrum. In this sense, ignoring of the high degree of neutrino spectrum nonequilibrium or, in other words, description of experimental effective neutrino spectrum by the equilibrium neutrino spectra of individual nuclides or their mixture can result in serious mistakes in fitting the experimental neutrino spectrum. Let us consider this in more detail.

\section{On Some Important Features of Alternative Treatment Procedure of KamLAND Experimental Data}

\subsection{Time Variation of the Reactor Antineutrino Flux and Upper Limit of Georeactor Power}

In the paper by KamLAND-collaboration [2], where the results of the second exposure (551.1 days) are analyzed, the original and very interesting method for determination of antineutrino rate suppression factor, which describes the degree of distortion of antineutrino spectrum, is presented. For that purpose the time variations of the reactor antineutrino flux assuming no antineutrino oscillation were estimated (see Figure 15(a)).

Analysis of the linear dependence of the observed $\tilde{v}_{e}$ event rate on the no-oscillation reactor $\tilde{v}_{e}$ flux and assumption that the slope can be interpreted as $\tilde{v}_{e}$ rate suppression factor and the intercept as the reactor-independent constant background rate (Figure 15(b)) are the
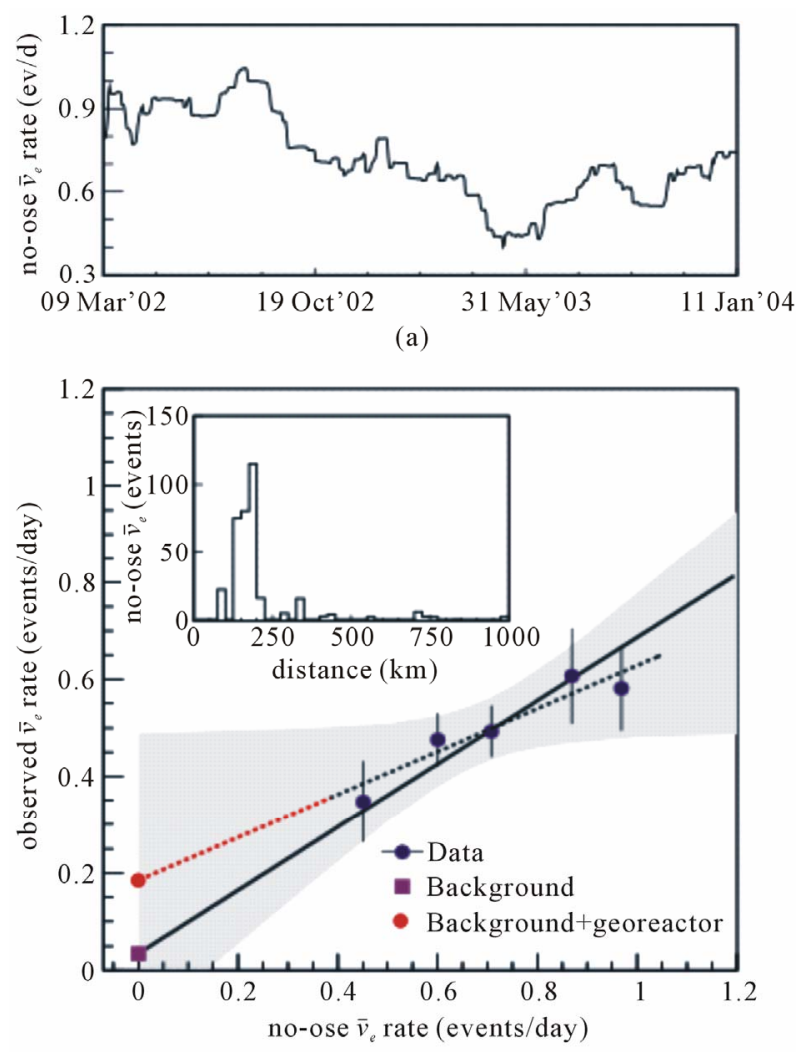

(b)

Figure 15. Adapted from [2]. (a) Estimated time variation of the reactor $\tilde{v}_{e}$ flux assuming no antineutrino oscillation. (b) Observed $\tilde{v}_{e}$ event rate versus no-oscillation reactor $\tilde{v}_{e}$ flux. Data points correspond to intervals of approximately equal $\tilde{\boldsymbol{v}}_{e}$ flux. The dotted black line is a fit [2]; the $90 \%$ C.L. is shown in gray [2]. The solid black line is a fit constrained to the expected background [2]. The dotted red line is a continuation of the dashed black line, the intercept of which in this case is equal to the sum of expected background and $\tilde{v}_{e}$

flux from unknown source (for example, a georeactor). The reactor distance distribution for $\tilde{v}_{e}$ events in the absence of oscillation is shown in the inset.

main points of this method. The solid black line in the Figure 15(b) is the linear KamLAND-fit $(90 \%$ C.L.) constrained to the expected background [2]. As analysis of this experiment shows, the value of this expected background corresponds to the sum of background contributions from different background nuclear reactions to the $\tilde{v}_{e}$ signal above $2.6 \mathrm{MeV}$ and is equal to $17.8 \pm 7.3$ events. Here it is important to note that to obtain such a fit, the authors of Ref. [2] use the strict assumption that "...the intercept is consistent with known background, but substantially larger backgrounds cannot be excluded; hence this fit does not usefully constrain speculative sources of antineutrinos such as a nuclear reactor at the Earth core [15] ...". In other words, being applied to the analysis of the observed $\tilde{v}_{e}$-spectra, this assumption is 
equivalent to acceptance of the so-called zero KamLAND-hypothesis, which a priori rules out the existence of nuclear georeactor or, at least, rejects the significant influence of an additional $\tilde{v}_{e}$ flux from the nuclear georeactor (even if it exists) on the observed $\tilde{v}_{e}$-spectra. In the last case, taking into account the parameters of mixing obtained within the framework of zero KamLAND-hypothesis, “... the predicted KamLAND rate for typical $3 \mathrm{TW}$ georeactor scenarios is comparable to the expected $17.8 \pm 7.3$ event background and would have minimal impact on the analysis of the reactor power dependence signal" [2].

On the other hand, the simple analysis of the dashed black line in Figure 15(b) shows that the alternative, i.e. nonzero, KamLAND-hypothesis, which recognizes the existence of nuclear georeactor with considerable heat power, has all the rights to exist. Note that the dotted black line is a fit [2], the $90 \%$ C.L. is shown in gray [2] and the dotted red line is our prolongation of the dashed black line, the intercept of which in this case is equal to the sum of expected background and $\tilde{v}_{e}$ flux from an unknown source.

Below we assume that the nuclear georeactor plays the role of unknown $\tilde{v}_{e}$ source. Let us estimate its heat power $W$. It will be recalled that for determination of reactor power in neutrino experiments, according to Equations (8)-(10) and under other conditions being equal, it is necessary to know 1) the location of georeactor, i.e. the distance between the georeactor and detector; 2) fuel composition and the corresponding equilibrium (or nonequilibrium $^{2}$ ) concentrations of fission products; 3 ) georeactor antineutrino spectrum uncertainty; 4) the survival probability $p$ or the neutrino oscillation parameters. Let us assume that for the nuclear georeactors with the heat power $W_{0} \sim 3 \mathrm{TW}$ (the zero KamLAND-hypothesis [2] and $W$ (the alternative nonzero KamLAND-hypothesis) the conditions 1) and 2) are identical. Then, taking into account that the intercepts and slopes of straight lines in Figure 15(b) corresponding to the solid black line (the zero KamLAND-hypothesis) and the dotted red line (the alternative nonzero KamLAND-hypothesis) are ${ }^{0} n_{v} \sim 0.32$, $\Sigma_{n_{v}} \sim 0.195$ and $p_{0} \sim 0.6, \quad p \sim 0.4$, respectively, it is easy to obtain the approximate estimation of the nuclear georeactor heat power $W$ within the framework of alternative nonzero KamLAND-hypothesis:

$$
\frac{{ }^{\Sigma} n_{v}}{{ }^{0} n_{v}}=\frac{p W+p_{0} W_{0}}{p_{0} W_{0}} \rightarrow W \sim 22 \mathrm{TW} .
$$

It is necessary to notice that the survival probability $p \sim 0.4$ is not characterized by the neutrino oscillation parameters computed for the zero hypothesis any more.

In our opinion, the given example, which shows some

${ }^{2}$ For the details of the calculations of nonequilibrium neutrino spectra see below (Section 6). details of the reactor antineutrino flux variations application, is extremely obvious and significant since it substantiates in a natural way the possibility of existence of the two alternative (but physically equal in rights) hypotheses for the interpretation of experimental KamLANDdata.

In this sense it is interesting to examine the results by Fogli et al. [51], who analyzed the KamLAND neutrino spectra in energy and time exactly for the second exposure [2]. They acted on the premise that "while the energy spectrum KamLAND events allows the determination of the neutrino oscillation parameters, the time spectrum can be used to monitor known and unknown neutrino sources". By using available monthly-binned data on event-by-event energies in KamLAND and on reactor powers in Japan, they performed a likelihood analysis of the neutrino event spectra in energy and time, and not only confirmed the results of KamLAND-collaboration, but also set the upper bound on hypothetical georeactor power in the framework of nonzero hypothesis $(W \leq 13$ TW at $95 \%$ C.L.).

Here a natural question arises: "Why do we obtain the different values of the neutrino oscillation parameters and upper bound on hypothetical georeactor power in comparison with Fogli et al. analyzing the same KamLAND-experiment within the framework of the same nonzero hypothesis?" Obviously it has nothing to do with the likelihood analysis ideology, which is clearly stated in the paper by Folgi [51] in detail, but rather is a conesquence of the completely different understanding of the hypothetical nuclear georeactor physical properties, some of which are discussed above (see derivation of Equation (12)). Let us demonstrate this.

In general, the KamLAND unbinned likelihood function $\mathcal{L}$ can be written as [1,2,51,52]

$$
\mathscr{L}=\mathscr{L}_{\text {rate }} \times \mathscr{L}_{\text {shape }} \times \mathscr{L}_{\text {syst }} .
$$

where the three factors embed information on the total event rate, on the spectrum shape and on the systematic uncertainties.

According to [51], we remain the second and third likelihood factors in Equation (19) unchanged and consider only the first likelihood factor, which can be written as (see also $[1,2,51,52])$ :

$$
\begin{aligned}
\mathscr{L}_{\text {rate }}= & \frac{1}{\sqrt{2 \pi} \sigma_{\text {rate }}} \\
& \exp \left[-\frac{1}{2}\left(\frac{N_{\text {theor }}\left(\delta m^{2}, \sin ^{2} \theta_{12} ; \alpha, \alpha^{\prime}, \alpha^{\prime \prime}\right)-N_{o b s}}{\sigma_{\text {rate }}}\right)^{2}\right]
\end{aligned}
$$

where $\left(\delta m^{2}, \sin ^{2} \theta_{12}\right)$ are the mass-mixing parameters, $\alpha$ is the systematic energy offset, $\alpha^{\prime}$ and $\alpha^{\prime \prime}$ are free 
(positive) parameters, $N_{o b s}$ is the total number of observed events and the total error is the sum of the statistical and systematic uncertainties

$$
\sigma_{\text {rate }}^{2}=N_{\text {theor }}+\left(s N_{\text {theor }}\right)^{2},
$$

where $s$ is a fraction of systematic uncertainty.

Here is the key moment which reveals the physical distinction between our nonzero hypothesis and nonzero hypothesis by Fogli et al. [51]. In general case, when the additional antineutrino source (i.e. a nuclear georactor) is taken into account within the framework of the nonzero hypothesis, it is also necessary to take into account the uncertainty of georeactor antineutrino spectrum. As is shown above (see Equation (12)), this uncertainty appears due to the change of fission cross-section of ${ }^{239} \mathrm{Pu}$ (which is the main component of nuclear fuel) with change of nuclear fuel temperature and, in particular, with change of temperature near the Earth solid core surface (see Figure 3). As a result, in the case of nonzero hypothesis Equation (21) must have, according to Equation (12), the following form:

$$
\begin{aligned}
& \sigma_{\text {rate }}^{2}=N_{\text {theor }}+\left[s\left(N_{\text {theor }}-N_{\text {grn }}\right)\right]^{2}+\left(s_{0} N_{\text {grn }}\right)^{2}, \\
& s_{0} \sim 1 \gg s,
\end{aligned}
$$

where $N_{\text {theor }}=N_{\text {jap }}+N_{\text {grn }}, N_{\text {Jap }}$ is the total number of events from Japanenese nuclear reactors, $N_{g r n}$ is the total number of events from nuclear georeactor, $s_{0}$ is a contribution from systematic uncertainty of the number of georeactor antineutrino.

It is obvious that Equation (22) in contrast to Equation (21) allows higher values of the nuclear georeactor heat power. This, in its turn, leads to a change in survival probability and, consequently, to a change in the neutrino oscillation parameters. In this sense, it is clear that even a very accurate account taken of the time variation of the reactor antineutrino flux (for example, monthly or even daily neutrino flux) and of another not less important features of antineutrino spectrum does not lead to a considerable change of the antineutrino survival probability (see Figure 15(b), solid black line), if the specific uncertainty of georeactor antineutrino spectrum is not taken into account as well. And vice versa, taking into account such a feature of georeactor antineutrino spectrum let us obtain the new restrictions on the georeactor heat power and corresponding values of the neutrino oscillation parameters (Figure 5) by likelihood analysis of the KamLAND energy spectrum (Figure 4) and minimization of corresponding $\chi^{2}$-function based on Equation (19).

Returning to the known KamLAND estimation of georeactor heat power, we would like to cite Ref. [4]: "The KamLAND-data, together with solar $v$ data, set an upper limit of $6.2 \mathrm{TW}\left(90 \%\right.$ C.L.) for a $\tilde{v}_{e}$ reactor source at the Earth center [15] assuming that the reactor produced a spectrum identical to that of a slow neutron artificial reactor". Although it does not evidently follow from the paper, we suppose that within the framework of likelihood analysis of the KamLAND neutrino spectra in energy and time the authors used the nonzero georeactor hypothesis by adding a $57^{\text {th }}$ reactor at $L=6400 \mathrm{~km}$ to the 56 Japanese nuclear power reactors. At the same time, the nontrivial properties of some components of nuclear fuel (for example, the ${ }^{239} \mathrm{Pu}$ fission cross-section (see Figure 3)) were not taken into account at all in this paper as well as in all other papers by KamLAND-collaboration. As is shown above, such a neglect of anomalous behavior of the ${ }^{239} \mathrm{Pu}$ fission cross-section at high temperatures (in the 2500 to $6000 \mathrm{~K}$ range (Figure 3)) implies, according to Equation (22), the ignoring of high uncertainty of georeactor antineutrino spectrum, which within the framework of maximum likelihood analysis will immediately cause the distortion of "true" values of reactor heat power and corresponding values of the neutrino oscillation parameters.

Finally it is worth mentioning that a nonstationary regime of nuclear georeactor operating, caused by the change of the ${ }^{239} \mathrm{Pu}$ fission rate (Equation (5)) mainly due to the strong variation of the ${ }^{239} \mathrm{Pu}$ fission cross-section (Equation (7)), which is a nonlinear function of medium temperature (see Figure 3), is the main reason of high uncertainty of georeactor antineutrino spectrum. Such a nonstationary regime generates yet another, quite deep and nontrivial problem, i.e. the so-called problem of nonequilibrium neutrino spectra. Rejection of this problem can lead to serious errors in fitting of the experimental neutrino spectra. Below we consider this in more detail.

\subsection{On the Reasons and Degree of the Antineutrino Spectra Nonequilibrium in KamLAND Experiments}

To describe the nuclear fuel antineutrino radiation, the nuclide equilibrium concentrations of fission-product mixture and, accordingly, equilibrium antineutrino spectra obtained for hypothetical infinite irradiation time, which provides a secular equilibrium of all without exception fission products, are traditionally used as a zero-order approximation.

On the other hand, it is obvious that the equilibrium approximation is not true for the non-stationary nuclear georeactor (Figure 14). Therefore, there is a question, how the strategy of calculation of effective neutrino spectra changes in this case and, in particular, how the resulting neutrino spectrum shape changes due to taking into account the nonequilibrium neutrino spectra instead of equilibrium neutrino spectra, which are used for stationary nuclear reactors.

As is known, a direct summation method of $\beta, v-$ 
spectra of individual nuclides $[42,43]$ composing the fission-product mixture with regard to the specific modes of fuel irradiation in a nuclear reactor, and proper total effective $\beta, v$-spectrum of the nuclear system $k$

$$
\rho^{k}(E)=\sum_{j} \lambda_{j} N_{j}^{k} \rho_{j}(E),
$$

are used as the calculation algorithm when passing from the $\beta$-spectrum to the antineutrino spectrum. Here $\lambda_{j}$ is the decay probability of $j^{\text {th }}$ nuclide; $N_{j}$ is the number of nuclei of $j^{\text {th }}$ nuclide in the system $k ; \rho_{j}(E)$ is the total $\beta, v$-spectrum of $j^{\text {th }}$ nuclide normalized by the nuclear decay:

$$
\rho_{j}(E)=\frac{K_{j}}{\sum_{i} v_{i, j}} \sum_{i} v_{i, j} \rho_{i, j}(E) .
$$

where $\lambda_{j} N_{j}$ is the activity of $j^{\text {th }}$ nuclide depending on irradiation mode (fuel initial composition, neutron flux density, fuel burn-up and other parameters influencing on accumulation of each $j^{\text {th }}$ nuclide); $K_{j}$ is the branching factor for $\beta$-decay channel, i.e. the number of $\beta$-particles per decay; $\rho_{i, j}(E)$ is the partial $\beta$-transition spectrum of $j^{\text {th }}$ nuclide; $v_{i, j}$ is the $\beta$-transition intensity of $j^{\text {th }}$ nuclide.

A priori knowledge (based on calculation or experimental estimation) of the initial concentration $N_{j}(t)$ of $j^{\text {th }}$ fissionable actinoid and the one-group integral neutron flux density $\Phi$ makes it possible to determine the accumulation of one or another $j^{\text {th }}$ fission product in the reactor core by solving the following system of kinetic equations ${ }^{3}$

$$
\begin{aligned}
\frac{\mathrm{d} N_{j}}{\mathrm{~d} t}= & -\lambda_{j} N_{j}-\left\langle\sigma_{c}\right\rangle_{j} \Phi N_{j}+\sum_{i=1}^{p}\langle\gamma\rangle_{i j}\left\langle\sigma_{f}\right\rangle_{i} \Phi N_{i} \\
& +\sum_{m=1}^{j-1} \lambda_{m j} N_{m}+\sum_{m=1}^{j-1}\left\langle\sigma_{c}\right\rangle_{m j} \Phi N_{m}
\end{aligned}
$$

which describes the time change of the $j^{\text {th }}$ nuclide concentration $N_{i}(t)$ in fission-product mixture at the initial condition $N_{j}(0)=N_{0 j}$ at the time $t$ linked with the activity of the $j^{\text {th }}$ nuclide in the following way $Q_{j}(t)=$ $\lambda_{j} \cdot N_{j}(t)$; index " $m$ " relates to precursor nucleus, $m<j$; $\langle\gamma\rangle_{i j}$ is the independent yield of the $j^{\text {th }}$ nuclide due to the fuel $i^{\text {th }}$ component fission averaged over the effective neutron spectrum; $\lambda_{m j}$ is the decay probability of $m^{\text {th }}$ nuclide into the $j^{\text {th }}$ nuclide due to $\beta$-, $\beta^{+}$-decay, electron capture, isomeric transition etc.; $\left\langle\sigma_{f}\right\rangle_{i}$ is the one-group fission cross-section for the $i^{\text {th }}$ fissionable actinoid;

\footnotetext{
${ }^{3}$ Note that the index $i$ changes in the range $1 \leq i \leq p$, and according to the known Russian catalogue of radioactive nuclides total $\beta, v$-spectra [42], the index $j$ changes in the range $1 \leq j \leq 1028$, i.e. the matter is a necessity to solve the enormous system of enchained differential equations. The method of solution of the system (25) based on the derivation of recurrence relations for $N_{j}(t)$ is in detail described in [53] and realized as the AFPA program package (Accumulation of Fission Products and Actinides) in terms of FORTRAN-IV.
}

$\left\langle\sigma_{c}\right\rangle_{i}$ is the one-group $(n, \gamma),(n, 2 n)$ reaction cross-section for $i^{\text {th }}$ nuclide; $\left\langle\sigma_{c}\right\rangle_{m j}$ is the $(n, \gamma),(n, 2 n)$ reaction cross-section for $m^{\text {th }}$ nucleus with $j^{\text {th }}$ nucleus formation.

Finding the time dependence of nuclide concentration of fission-product mixture (see (25)) is a sufficiently labor-consuming problem, and its solution depends on the specific conditions of fuel irradiation, i.e. the time dependence of neutron flux density, neutron flux spectral composition and also the initial composition of the fuel [42, 43]. Therefore the exact solution of the kinetic system of Equations (25) becomes practically unachievable in the study of fission-product build-up in nonstationary nuclear reactors with nonconstant or in general unknown parameters. All said above applies in full measure to the nonstationary nuclear georeactor, the thermal power evolution of which is shown in Figure 14. Moreover, in this case a situation is aggravated by the fact that the ${ }^{239} \mathrm{Pu}$ fission cross-section (the main active component of georeactor nuclear fuel) is a strongly nonlinear magnitude, which grows by the power law in the temperature range $3000-5000 \mathrm{~K}$ (Figure 3), which is typical for the near-surface layers of the Earth solid core.

How much is better, when the reactor is stationary. In this case, the left-hand sides of the system of the Equations (25) can be set equal to zero, and the system itself transforms into the system of linear algebraic equations, the solution of which (the so-called equilibrium nuclide concentrations of fission product mixture) does not depend on initial conditions and irradiation time. The obtained equilibrium nuclide concentrations of fission product mixture make it possible (according to Equation (23) to determine equilibrium partial and total neutrino spectra, which are usually used for description of effective neutrino spectra of stationary neutrino sources and, in particular, stationary nuclear reactors.

Finally, returning to the analysis of concrete KamLAND neutrino spectra, it is necessary to state that within the framework of georeactor hypothesis an integral fraction of reactor geoantineutrino is sufficiently great and makes up almost the half of integral fraction of antineutrinos produced by all Japanese reactors in the KamLAND-experiment (see Figures 4 and 13). It means that the non-equilibrium property inherent to the reactor geoneutrino spectrum is not only delegated to the KamLAND experimental neutrino spectrum to a considerable extent, but plays a dominant role in natural distortion of this spectrum with respect to the KamLAND theoretical neutrino spectrum, which is based on the ideology of equilibrium neutrino spectra.

The question arises, to which degree this non-equilibrium influences on the effective neutrino spectrum shape in the general case and, for example, in KamLAND-experiments. As the numerous test experiments related to the nuclear fuel irradiation under unstable conditions 
show [42,53], the non-equilibrium effect manifests itself as the observed distortion of some pieces of the nonequilibrium effective spectrum (with respect to an analogical equilibrium neutrino spectrum), the location of which in spectrum energy coordinates is completely predetermined by the time dependence of neutron flux density, neutron flux spectral composition and initial composition of the fuel $[42,53]$.

On the other hand, the analysis of experimental KamLAND-data obtained over the years 2002-2004, 20022007 and 2002-2009 shows that in all considered cases the number of recorded events in the fifth $\left(E_{5}=2.8 \mathrm{MeV}\right)$ and ninth $\left(E_{9}=4.5 \mathrm{MeV}\right)$ energy bins of experimental neutrino KamLAND-spectra differs substantially from the similar data obtained by fitting or, in other words, theoretical equilibrium neutrino KamLAND-spectra (see Figure 1 in [4], Figure 1 in [5] and also Figures 4 and 13). At the same time, the number of recorded events in the fifth bin is always substantially less than in the fifth bin of theoretical neutrino KamLAND-spectrum, whereas an opposite situation is observed for the ninth bin. It is the so-called problem of 5 and 9 energy bins of neutrino KamLAND-spectra, which, in our opinion, is caused not only by detection statistics, but is mainly a manifestation of substantial non-equilibrium of neutrino spectra. According to $[42,43]$, the amount of non-equilibrium effect, i.e. the difference between calculated equilibrium neutrino spectra and corresponding experimental non-equilibrium neutrino spectra can reach $10 \%-15 \%$ and more.

At last, it is necessary to remind that in this paper all theoretical neutrino spectra (Figures 2, 4, 9 and 13) are built using the ideology of equilibrium spectra. Within the framework of georeactor hypothesis such an approach is reasonable, because the possible high degree of non-equilibrium of experimental neutrino spectra, which manifests itself, for example, as the so-called problem of the 5 and 9 energy bins of neutrino KamLAND-spectra, is effectively compensated by the high, but reasoned degree of uncertainty of theoretical neutrino spectra.

\subsection{Geological (Magnetic) Time-Scale and Time Evolution of the Nuclear Georeactor Power}

Within the framework of the alternative hypothesis we obtained the time evolution of the average georeactor heat power over the period of 2002-2009 (Figure 14), which shows that the average georeactor heat power $W_{\mathrm{t}}$ drops sharply from 50 TW to 5 - 7 TW in KamLANDexperiments over the periods of 2002-2004, 2005-2007 and 2008-2009. Here a natural question arises: "What does such a dynamics reflect or what physical mechanism causes such a dynamics?" In other words, is it a manifestation of some unknown physics or a trivial consequence of the "... luckily guessed calculation rules which do not represent the true nature of things" [54]. Below we try to give a simple and physically clear interpretation of the possible fundamental mechanism and its impact on the temporal dynamics of the nuclear georeactor heat power.

It is known that in spite of its long history, the nature of the energy source maintaining a convection in the Earth liquid core or, more exactly, the mechanism of the magneto-hydrodynamic dynamo (MHD) generating the terrestrial magnetic field has no clear and unambiguous physical interpretation so far $[26,55]$. The problem is aggravated by fact that none of candidates for an energy source of the geomagnetic field [55] (secular cooling due to the heat transfer from the core to the mantle, internal heating by radiogenic isotopes, e.g. ${ }^{40} \mathrm{~K}$, latent heat due to the inner core solidification, compositional buoyancy due to the ejection of light element at the inner core surface) can not in principle explain one of the most remarkable phenomena in solar-terrestrial physics - a strong (inverse) correlation between the temporal variations of magnetic flux in the tachocline zone (the bottom of the Sun convective zone) $[56,57]$ and the Earth magnetic field ( $Y$-component) ${ }^{4}$ [59] (Figure 16).

At the same time, supposing that the transversal (radial) surface area of tachocline zone, through which a magnetic flux propagates, is constant in the first approximation, one can assume that magnetic flux variations describe also the temporal variations of magnetic field in the solar tachocline zone. Thus, Figure 16 demonstrates the mirror correlation between the temporal variations of magnetic field in the solar tachocline zone and the Earth magnetic field ( $Y$-component).

To obtain such visual correlations we used the so-called moving interval averaging algorithm. The procedure of averaging the series $\left\{x_{n}\right\}$ in order to derive the averaged (by means of the moving interval of $N$ points) series $\left\{\langle x\rangle_{n}^{N}\right\}$ was performed using the following expression:

$$
\langle x\rangle_{n}^{N}=\frac{1}{N}\left(\sum_{i=1}^{(N-1) / 2}\left(x_{n-i}+x_{n+i}\right)+x_{n}\right),
$$

where $N=2 k-1 \geq 3$.

\footnotetext{
${ }^{4}$ Note that the strong (inverse) correlation between the temporal variations of magnetic flux in the tachocline zone and the Earth magnetic field ( $Y$-component) are observed (Figure 16) only for experimental data obtained at the observatories where the temporal variations of declination $(\delta D / \delta t)$ or the closely associated east component $(\delta Y / \delta t)$ are directly proportional to the westward drift of magnetic features [58]. This condition is very important for understanding of physical nature of indicated above correlation, since it is known that the motions of the top layers of the Earth core are responsible for most magnetic variations and, in particular, for the westward drift of magnetic features seen on the Earth surface on the decade time scale. Europe and Australia are in [58]).
} 


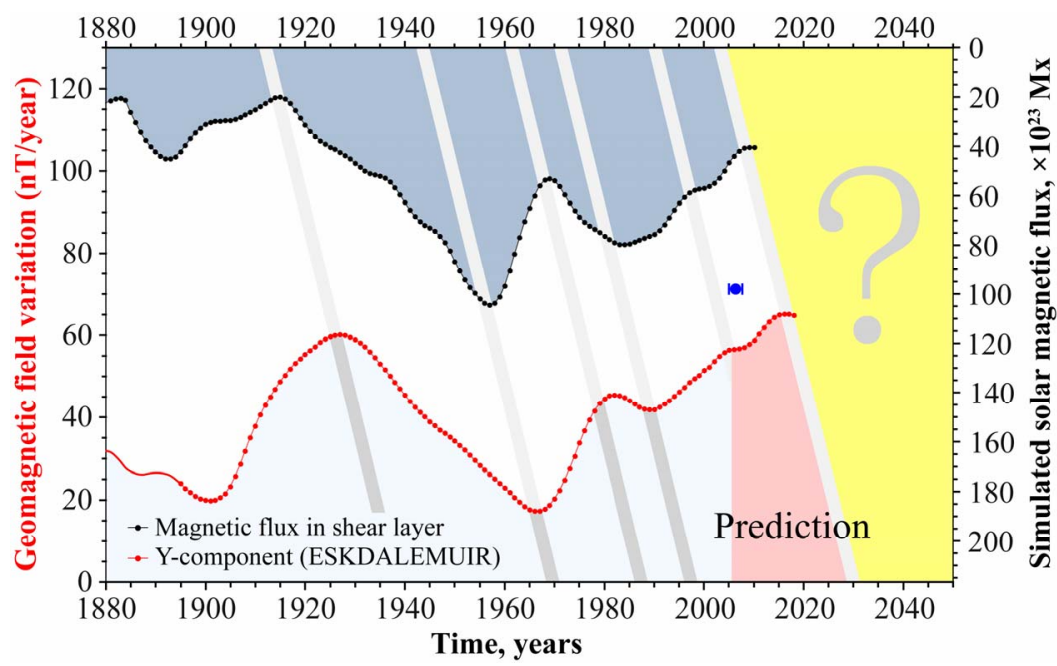

Figure 16. Time evolution of the magnetic flux variations at the bottom (tachocline zone) of the solar convective zone ((black dotted line, see Figure 17), variations of the nuclear georeactor thermal power (blue point with bars), geomagnetic field secular variations ( $Y$-component, $n T / y e a r$, red dotted line) [59] and prediction (green dotted line) [59]. All curves are smoothed by sliding intervals in 5 and 11 years. The pink area is the prediction region.

where $k$ is the positive integer.

The smoothed curve of the magnetic flux variations in the solar tachocline zone, which is shown in Figure 17(c) (black dotted line), demonstrates the result of such an averaging of the initial curve (red fill area in Figure 17(c)) by the two sliding intervals in $N=5$ and $N=11$ years. Physical sense of such a double averaging consists in the "soft" removing of the 11-year solar cycle influence and obtaining of the so-called amplitude-modulated representation of magnetic flux variations in the solar tachocline zone.

It follows from Figure 16 that the degree of averaging effect on time sample of the variations of solar magnetic field and the Earth magnetic field ( $Y$-component) sharply differs in the degree of their smoothing. In our opinion, this is caused by a considerable delay $(10-12$ years $)$ of the variations of terrestrial magnetic field ( $Y$-component) with respect to the solar magnetic field variations, during which the intermediate deep terrestrial processes controlled by the solar power pacemaker not only activate and maintain the Earth magnetic field, but also smooth out some fine "details" of the solar power pacemaker influence (Figure 17). Below we consider the physical mechanism of the one possible intermediate deep terrestrial process by virtue of which a future candidate for an energy source of the Earth magnetic field must play not only the role of a natural trigger of solar-terrestrial connection, but also directly generate the solar-terrestrial magnetic correlation on its own.

We assume that the temporal variation of soliton-like nuclear georeactor heat power can be a global energy cause of such a fundamental geophysical phenomenon as the variations of terrestrial magnetic field ( $Y$-component).

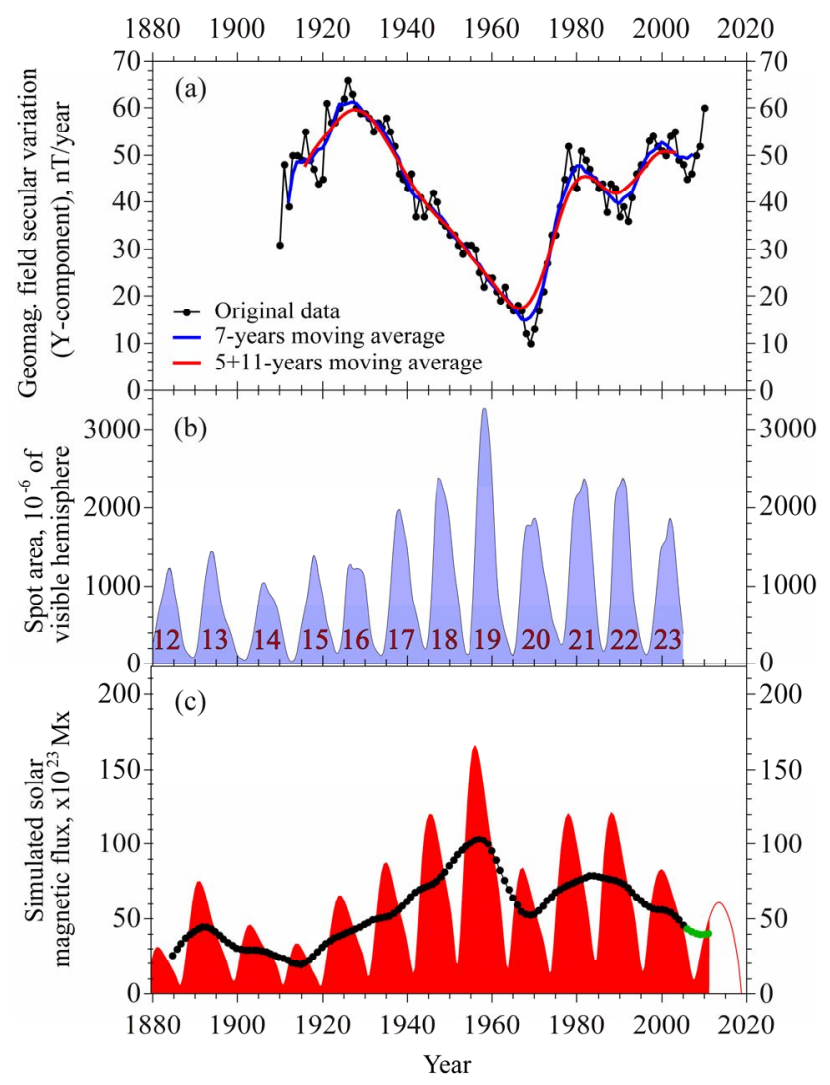

Figure 17. Time evolution of (a) geomagnetic secular variations ( $Y$-component, nT/year) [59], (b) observed sunspot area for cycles 12 - 23 [57], (c) integral from $0^{\circ}-45^{\circ}$ latitude of simulated toroidal magnetic flux in bottom shear layer (red shadow zone) for cycles 12 - 23 [57], plus forecast for cycle 24 [57]. Black curves are smoothed by sliding intervals in 5 and 11 years. The green dotted line is the prediction region. 
If it is truth, it must be the Solar magnetic field variations that "drive" or cause the temporal variations of the nuclear georeactor heat power, which, according to our hypothesis, is the energy source of the Earth magnetic field and, consequently, its variations. One of possible mechanisms generating strong (negative) correlation between the terrestrial magnetic field and solar magnetic field (see Figure 16) can be so-called axion mechanism of solar dynamo-geodynamo connection [60].

The essence of this mechanism is that the total energy of ${ }^{57} \mathrm{Fe}$-axions produced mainly in the Sun core is modulated at first by the magnetic field of the solar convective zone (due to the inverse coherent Primakoff effect [60]) and after that is resonance absorbed by ${ }^{57} \mathrm{Fe}$ in the Earth core. In other words, the higher the solar magnetic field, the greater the number of axoins is converted (by the inverse Primakoff effect) into $\gamma$-quanta, the smaller the number of axoins reach the Earth and are absorbed in the Earth core, and vice versa.

It results in the fact that the variations of ${ }^{57} \mathrm{Fe}$-axion intensity play the role not only of heat source, which changes the temperature of the Earth core, but also the modulator of nuclear georeactor thermal power, because the medium temperature in the range 2500 - $6000 \mathrm{~K}$ modulates the value of the ${ }^{239} \mathrm{Pu}$ fission cross-section (Figure 3). In other words, the solar axions mechanism not only explains the nature of heat source in Earth liquid core, which plays the role of the modulator of nuclear georeactor thermal power, but in a natural way explains the cause of experimentally observed strong inverse correlation (Figure 16) between the magnetic field of the solar convective zone and the Earth magnetic field ( $Y$ component).

From Figure 16 it follows that the variation of the Earth magnetic field ( $Y$-component) lags behind the variation of the solar magnetic field about 10 - 12 years. On the other hand, it is known that a magnetic signal predetermined by an extremum of drift velocity of eccentric dipole of the Earth core lags and therefore is detected on the Earth surface $5-7$ years late $[61,62]$. Within the framework of georeator hypothesis this means that the temporal variation of magnetic field energy $W_{S E}$ on the Earth surface also has the delay of $5-7$ years with respect to the temporal variation of magnetic field energy $W_{\text {core }}$ of the Earth liquid core which, in its turn, is formed with the delay with respect to the temporal variations of the nuclear georeactor thermal power $W$. At the same time, since $W_{\text {core }} \sim B^{2}$, where $\boldsymbol{B}$ is the magnetic induction vector, we can obtain from the obvious relation $W_{\text {core }}$ $\sim W$ that

$$
B \sim \sqrt{W} .
$$

On the other hand, we have found the sampling of values for the nuclear georeactor thermal power (Table
1), which is obtained by the experimental KamLAND data handling over the years of 2002-2009. This sampling contains three averaged values: 50.2 TW over the years of 2002-2004, 21.1 TW over the years of 20052007 and 7.3 TW over the years of 2008-2009. Due to delay of temporal variation of the nuclear georeactor thermal power with respect to the variation of the Earth magnetic field ( $Y$-component) it is obvious that to smooth out the influence of background processes (the variations of the Earth liquid core temperature, nuclear fuel composition etc.) accompanying geodynamo operation, it is necessary to average the sampling of values of the nuclear georeactor thermal power by a sliding time interval with the length of order of a delay time, i.e. $N=$ 5 - 7 years. It is rather easy to show that, using a maximum possible sliding interval with $N=7$ transforms this sampling composed of the 7 virtual annual values over the years of 2002-2009 into the sampling which contains only one term characterizing the average nuclear georeactor thermal power

$$
\langle W\rangle_{4}^{7} \sim 30 \mathrm{TW}
$$

It is obvious that this single term of new sampling corresponds to the year 2006. If, according to the solar axion mechanism, we assume the existence of a strong (inverse) correlation between the variation of the Earth magnetic field and the value $\sqrt{W}$ (see Equation (27)) and also take into account the delay time ( $5-7$ years) of the variation of $\sqrt{W}$ with respect to the variation of the solar magnetic field, it is easy to find the coordinates of nuclear georeactor in Figure 16. The value of $\sqrt{W}$ is at the intersection of vertical line $t=2006$ and the slanting grey line passing through the extreme point $(t=2001)$ on the curve of variation of the solar magnetic field. Let us remind that the slope of the grey straight line in Figure 16 is the effect of delay under the conditions of strong (inverse) correlation of the solar magnetic field and the Earth magnetic field ( $Y$-component).

Note that all the future measurements of annual variations of neutrino flux in the KamLAND and Borexino experiments will generate new theoretical data describing the variations of nuclear georeactor thermal power. If these variations smoothed by moving-average process will correlate with the variations of the solar magnetic field and variations of the Earth magnetic field (Y-component), the georeactor hypothesis will obtain another strong indirect confirmation.

Finally, we would like to remind that within the framework of georeactor hypothesis, a forecast of the abovementioned fundamental geophysical processes behavior, which have a common cause (the temporal variations of magnetic field in the solar tachocline zone), is possible only up to a corresponding event horizon predetermined by delay time of the nuclear georeactor power variation 
(5 - 7 years) or magnetic Y-field of the Earth (10 - 12 years) with respect the magnetic field in the solar tachocline zone. It is obvious that such a delay effect makes it possible to predict the behavior of the Earth magnetic field ( $Y$-component) reliably by experimental observation of georeactor antineutrino, the variations of which characterize the variations of nuclear georeactor thermal power.

\section{Conclusions}

We should note that although the nuclear georeactor hypothesis we used for the interpretation of the KamLAND-experiment seems to be very effective, it can be considered only as a possible alternative variant for describing the KamLAND experimental data. Only a direct measurements of the geoantineutrino spectrum in the energy range $>3.4 \mathrm{MeV}$ in the future underground or submarine experiments will finally resolve the problem of the natural georeactor existence and will make it possible to determine the "true" values of reactor antineutrino oscillation parameters. At the same time, the solution of the direct and the inverse problems of the remote neutrino-diagnostics for the intra-terrestrial processes which is essential to obtain the pure geoantineutrino spectrum and to determine a correct radial profile of the $\beta$-sources in the Earth interior $[43,63]$ will undoubtedly help us solve the problem of a natural nuclear reactor existence on the boundary of the liquid and solid phases of the Earth core.

In the second part of this paper [46] we will consider some properties of those fundamental geophysical phenomena, which must be observed directly under terrestrial conditions, if a georeactor hypothesis is true and the nuclear georeactor exists.

\section{REFERENCES}

[1] K. Eguchi, et al., "First Results from KamLAND: Evidence for Reactor Antineutrino Disappearance," Physical Review Letters, Vol. 90, No. 2, 2003, pp. 041801-041805. doi:10.1103/PhysRevLett.90.021802

[2] T. Araki, et al., "Measurement of Neutrino Oscillation with KamLAND: Evidence of Spectral Distortion," Physical Review Letters, Vol. 94, No. 8, 2005, pp. 08180110818015. doi:10.1103/PhysRevLett.94.081801

[3] T. Araki, et al., "Experimental Investigation of Geologically Produced Antineutrinos with KamLAND," Nature, Vol. 436, No. 7050, 2005, pp. 499-503.

doi:10.1038/nature 03980

[4] S. Abe, et al., "Precision Measurement of Neutrino Oscillation Parameters with KamLAND," Physical Review Letters, Vol. 100, No. 22, 2008, pp. 2218031-2218035. doi:10.1103/PhysRevLett.100.221803

[5] A. Gando, et al., "Enhanced Constraints on $\theta_{13}$ from a Three-Flavor Oscillation Analysis of Reactor Antineutri- nos at KamLAND," Physical Review D, Vol. 83, 2011, Article ID: 052002.

[6] P. K, Kuroda, "On the Nuclear Physical Stability of the Uranium Minerals," Journal of Chemical Physics, Vol. 25, No. 4, 1956, pp. 781-782. doi:10.1063/1.1743058

[7] P. K. Kuroda, "On the Infinite Multiplication Constant and the Age of the Uranium Minerals," Journal of Chemical Physics, Vol. 25, No. 6, 1956, pp. 1295-1296. doi:10.1063/1.1743220

[8] P. K. Kuroda, "Nuclear Fission in the Early History of the Earth," Nature, Vol. 187, 1960, pp. 36-38. doi:10.1038/187036a0

[9] G. A. Cowan, "A Natural Fission Reactor," Scientific American, Vol. 235, No. 1, 1976, pp. 36-47. doi:10.1038/scientificamerican0776-36

[10] A. P. Meshik, C. M. Honenberg and O. V. Pravdivtseva, "Record of Cycling Operation of the Natural Nuclear Reactor in the Oklo/Oklebondo Area in Gabon," Physical Review Letters, Vol. 93, No. 18, 2004, pp. 182302182305. doi:10.1103/PhysRevLett.93.182302

[11] R. B. Driscoll, "Nuclear Disruption of a Planet with Convective Outer Core," Bulletin of the American Physical Society, Ser II, Vol. 33, 1988, pp. 1031-1037.

[12] J. M. Herndon, "Nuclear Fission Reactors as Energy Sources for the Giant Outer Planets," Naturewissensch, Vol. 79, No. 1, 1992, pp. 7-14. doi:10.1007/BF01132272

[13] J. M. Herndon, "Feasibility of a Nuclear Fission Reactor at the Center of the Earth as Energy Source for the Geomagnetic Field," Journal of Geomagnetism and Geoelectricity, Vol. 45, No. 5, 1993, pp. 423-427. doi:10.5636/jgg.45.423

[14] D. F. Hollenbach and J. M. Herndon, "Deep-Earth Reactor: Nuclear Fission, Helium, and the Geomagnetic Field," Proceedings of the National Academy of Sciences USA, Vol. 98, No. 20, 2001, pp. 11085-11090. doi: $10.1073 /$ pnas. 201393998

[15] J. M. Herndon, "Nuclear Georeactor Origin of the Oceanic Basalt, ${ }^{3} \mathrm{He} /{ }^{4} \mathrm{He}$, Evidence and Implications," Proceedings of the National Academy of Sciences USA, Vol. 100, No. 6, 2003, pp. 3047-3050. doi: $10.1073 /$ pnas. 0437778100

[16] V. F. Anisichkin, A. A. Bezborodov and I. R. Suslov, "Chain Fission Reactions of Nuclides in the Earth Core during Billions Years," Atomic Energy, Vol. 98, No. 5, 2005, pp. 370-379.

[17] V. D. Rusov, et al., "Geoantineutrino Spectrum and Slow Nuclear burning on the Boundary of the Liquid and Solid Phases of the Earth Core," Journal of Geophysical Research, Vol. 112, No. B9, 2007, Article ID: B09203. doi:10.1029/2005JB004212

[18] V. F. Anisichkin, A. A. Bezborodov and I. R. Suslov, "Georeactor in Earth," Transport Theory and Statistical Physics, Vol. 37, No. 5-7, 2008, pp. 624-633. doi: $10.1080 / 00411450802515817$

[19] R. J. de Meijer and W. van Westrenen, "Assessing the Feasibility and Consequences of Nuclear Georeactors in Earth Core-Mantle Boundary Region," South African Journal of Science, Vol. 104, No. 3-4, 2008, pp. 111-118. 
[20] X. Z. Bao, "Distribution of U and Th and Their Nuclear Fission in the Outer Core of the Earth and Their Effects on the Geodynamics," Geological Review, Vol. 45, No. 1, 1999, pp. 82-92.

[21] D. L. Anderson, “The Helium Paradoxes," Proceedings of the National Academy of Sciences USA, Vol. 95, No. 9, 1998, pp. 4822-4827. doi:10.1073/pnas.95.9.4822

[22] D. L. Anderson, "The Statistics and Distribution of Helium in the Mantle," International Geology Review, Vol. 42, No. 4, 2000, pp. 289-311. doi:10.1080/00206810009465084

[23] D. L. Anderson, "Helium: Fundamental Models," 2003, pp. 6-7. http://www.mantleplumes.org/HeliumFundamentals.html

[24] H. M. Gonnermann and S. Mukhopadhyay, "Preserving Noble Gases in Convective Mantle," Nature, Vol. 459, No. 7246, 2009, pp. 560-564. doi:10.1038/nature08018

[25] T. Lay, et al., "A Post-Perovskite Lens and D" Heat Flux beneath the Central Pacific," Science, Vol. 314, No. 5803, 2006, pp. 1272-1276. doi:10.1126/science. 1133280

[26] S. L. Butler, W. R. Peltier and S. O. Costin, "Numerical Models of the Earth Thermal History: Effects of InnerCore Solidification and Core Potassium," Physics of the Earth and Planetary Interiors, Vol. 152, No. 1-2, 2005, pp. 22-42. doi:10.1016/j.pepi.2005.05.005

[27] V. D. Rusov, et al., "Galactic Cosmic Rays-Clouds Effect and Bifurcation Model of the Earth Global Climate. Part 2. Comparison of the Theory with Experiment," Journal of Atmospheric and Solar (Terrestrial Physics), Vol. 72, No. 5-6, 2010, pp. 389-397.

[28] L. P. Feoktistov, "From the Past towards the Future: from the Hopes of Bomb to the Safe Reactor," Publication of RFNC-Anrisph, Snezhinsk, 1998, p. 326.

[29] S. M. Feinberg, "Discussion Content," International Conference on the Peaceful Uses for Atomic Energy, Vol. 9, No. 2, 1958, p. 447.

[30] L. P. Feoktistov, "Neutron-Fissioning Wave," Reports of Academy of Sciences of the USSR, Vol. 309, 1989, pp. 864-867.

[31] E. Teller, M. Ishikawa and L. Wood, "Completely Automated Nuclear Reactors for Long-Term Operation," Proceedings of Frontiers in Physical Symposium Joint American Physical Society and American Association of Physics Teachers Texas Meeting, Lubbock, 1995.

http://www-phys.llnl.gov/adv_energy_src/ICENES96.html

[32] V. D. Rusov, E. P. Linnik, V. A. Tarasov, T. N. Zelentsova, I. V. Sharph, V. N. Vaschenko, S. I. Kosenko, M. E. Beglaryan, S. A. Chernezhenko, P. A. Molchinikolov, S. I. Saulenko and O. A. Byegunova, "Traveling Wave Reactor and Condition of Existence of Nuclear Burning Soliton-Like Wave in Neutron-Multiplying Media," Energies, Vol. 4, No. 9, 2011, pp. 1337-1361.

[33] D. N. Kracnoshchekov, P. V. Kaazik and V. M. Ovtchinnikov, "Seismological Evidence for Mosaic Structure of the Surface on the Earth Inner Core," Nature, Vol. 435, No. 7041, 2005, pp. 483-487. doi:10.1038/nature03613

[34] X. S. Bao and R. A. Secco, "U Solubility in Earth's Core," 2006, in press.
[35] S. T. Dye, "Neutrino Mixing Discriminates Geo-Reactor Models," Physics Letters B, Vol. 679, No. 1, 2009, pp. 15-18. doi:10.1016/j.physletb.2009.07.010

[36] G. Bellini, et al., "Observation of Geo-Neutrinos," Physics Letters B, Vol. 687, No. 4-5, 2010, pp. 299-304. doi:10.1016/j.physletb.2010.03.051

[37] A. M. Hofmeister and R. E. Criss, "Earth Heat Flux Revised and Linked Ti Chemistry," Tectonophysics, Vol. 395, No. 3-4, 2005, pp. 159-177. doi:10.1016/j.tecto.2004.09.006

[38] H. N. Pollack, S. J. Hunter and J. R. Johnson, "Heat Flow from Earth Interior: Analysis of the Global Data Net," Reviews of Geophysics, Vol. 31, No. 3, 1993, pp. 267-280. doi:10.1029/93RG01249

[39] D. L. Anderson, "Energetics of the Earth and the Missing Heat Mystery,” Elsevier Ltd., Oxford, 2007. www.mantleplumes.org/Energetics.html

[40] A. P. Van den Berg and D. Yuen, "Delayed Cooling of the Earth Mantle Due to Variable Thermal Conductivity and Formation of a Low Conductivity Zone," Earth and Planetary Science Letters, Vol. 199, No. 3-4, 2002, pp. 403-413. doi:10.1016/S0012-821X(02, pp. 00531-9

[41] A. P. Van den Berg, D. Yuen and V. Strinbach, "The Effects of Variable Thermal Conducnivity on Mantle Heat Transfer," Geophysical Research Letters, Vol. 28, No. 5, 2002, pp. 875-878. doi:10.1029/2000GL011903

[42] V. G. Aleksankin, et al., "Beta- and Antineutrino Nuclear Radiations," Energoatomizdat, Moscow, 1989, p. 312.

[43] V. D. Rusov, V. A. Tarasov and D. A. Litvinov, "Reactor Antineutrino Physics," URSS, Moscow, 2008, 430 p.

[44] B. Aharmim, et al., "Independent Measurement of the Total Active ${ }^{8}$ B Solar Neutrino Flux Using an Array of ${ }^{3} \mathrm{He}$ Proportional Counters at the Sudbury Neutrino Observatory," Physical Review Letters, Vol. 101, No. 11, 2008, pp. 1113011-1113015. doi:10.1103/PhysRevLett.101.111301

[45] V. M. Hamza, R. R. Cardoso and C. F. Ponte Neto, "Spherical Harmonic Analysis of Earth's Conductive Heat Flow," International Journal of Earth Sciences, Vol. 97, No. 2, 2008, pp. 205-226. doi:10.1007/s00531-007-0254-3

[46] V. D. Rusov, et al., "The KamLAND-Experiment and Soliton-Like Nuclear Georeactor. Part 2. Fundemental Geophysical Consequences," in preparation.

[47] G. Bellini, et al., "Observation of Geo-Neutrinos," Physics Letters B, Vol. 687, No. 4-5, 2010, pp. 299-304. doi:10.1016/j.physletb.2010.03.051

[48] T. Schwetz, M. Tortola and J. W. F. Valle, "Three-Flavor Neutrino Oscillation Update," New Journal of Physics, Vol. 10, No. 11, 2008, Article ID: 113011. doi:10.1088/1367-2630/10/11/113011

[49] H. F. J. Corr and V D. J. aughan, "A Recent Volcanic Eruption beneath the West Antarctic Ice Sheet," Nature Geoscience, Vol. 1, 2008, pp. 122-125. doi:10.1038/ngeo106

[50] Source. http://pubs.usgs.gov/gip/dynamic/world_map.html

[51] G. L. Fogli, E. Lisi, A. Palazzo and A. M. Rotunno, "KamLAND Neutrino Spectra in Energy and Time: Indi- 
cation for Reactor Poer Variations and Constraints of the Georeactor," Physics Letters B, Vol. 623, No. 1-2, 2005, pp. 80-92. doi:10.1016/i.physletb.2005.07.064

[52] T. Schvetz, "Variations on KamLAND: Likelihood Analysis and Frequentist Confidence Regions," Physics Letters B, Vol. 577, No. 3-4, 2003, pp. 120-128. doi:10.1016/j.physletb.2003.10.024

[53] V. M. Kolobashkin, P. M. Rubtsov, P. A. Ruzhanskiy, et al., "Calculated Characteristics of Irradiated Nuclear Fuel," Energoatomizdat, Moscow, 1983.

[54] V. V. Anisovitch, M. N. Kobrinsky, Y. Niri and Y. Shabelsky, "Additive Quark Model of Hadrons," Physics Uspehi, USSR, Vol. 144, 1984, p. 553.

[55] B. A. Buffet, "The Thermal State of Earth Core," Science, Vol. 299, No. 5613, 2003, pp. 1675-1677. doi:10.1126/science. 1081518

[56] M. Dikpati, G. de Toma and P. A. Gilman, "Polar Flux, Cross-Equatorial Flux, and Dynamo-Generated Tachocline Toroidal Flux as Predictors of Solar Cycles," The Astrophysics Journal, Vol. 675, No. 1, 2008, p. 920. doi:10.1086/524656

[57] M. Dikpati, "Predicting Cycle 24 Using Various DynamoBased Tools," Annales Geophysicae, Vol. 26, No. 2, 2008, pp. 259-267. doi:10.5194/angeo-26-259-2008

[58] "Data of the Observatory Eskdalemuir, (England)," World
Data Centre for Geomagnetic (Edinburg), 2007. http://www.geomag.bgs.ak.uk./gifs/annual_means.shtml

[59] J.-L. Le Mouel, T. R. Madden, J. Ducruix and V. Courtillot, "Decade Fluctuations in Geomagnetic Westward Drift and Earth Rotation," Nature, Vol. 290, 1981, pp. 763-765. doi:10.1038/290763a0

[60] V. D. Rusov, E. P. Linnik, K. Kudela, S. C. Mavrodiev, I. V. Sharh, T. N. Zelentsova, M. E. Beglaryan, V. P. Smolyar and K. K. Merkotan, "Axion Mechanism of the Sun Luminosity and Solar Dynamo-Geodynamo Connection," in press.

[61] E. H. Vestin and A. B. Kahle, "The Westward Drift and Geomagnetic Secular Change," Geophysical Journal of the Royal Astronomical Society, Vol. 15, No. 1-2, 1968, pp. 29-37. doi:10.1111/j.1365-246X.1968.tb05743.x

[62] R. Hide, D. H. Boggs and J. O. Dickey, "Angular Momentum Flucntuations within the Earth's Liquid Core and Torsional Oscillations of the Core-Mantle System," Geophysical Journal International, Vol. 143, No. 3, 2000, pp. 777-786. doi:10.1046/j.0956-540X.2000.01283.x

[63] V. D. Rusov, T. N. Zelentsova, V. A. Tarasov and D. A. Litvinov, "Inverse Problem of Remote Neutrino Diagnostic of Intrareactor Processes," Journal of Applied Physics, Vol. 96, No. 3, 2004, pp. 1734-1739. 